\title{
Multiple Antenna Techniques
}

\author{
Han-Kui Chang, Meng-Lin Ku, Li-Wen Huang and Jia-Chin Lin \\ Department of Communication Engineering, National Central University, Taiwan, \\ R.O.C.
}

\section{Introduction}

Recent developed information theory results have demonstrated the enormous potential to increase system capacity by exploiting multiple antennas. Combining multiple antennas with orthogonal frequency division multiplexing (OFDM) is regarded as a very attractive solution for the next-generation wireless communications to effectively enhance service quality over multipath fading channels at affordable transceiver complexity. In this regard, multiple antennas, or called multiple-input multiple-output (MIMO) systems, have emerged as an essential technique for the next-generation wireless communications. In general, an MIMO system has capability to offer three types of antenna gains: diversity gains, multiplexing gains and beamforming gains. A wide variety of multiple antennas schemes have been investigated to achieve these gains, while some combo schemes can make tradeoffs among these three types of gains. In this chapter, an overview of multiple antenna techniques developed in the past decade, as well as their transceiver architecture designs, is introduced. The first part of this chapter covers three kinds of diversity schemes: maximum ratio combining (MRC), space-time coding (STC), and maximum ratio transmission (MRT), which are commonly used to combat channel fading and to improve signal quality with or without channel knowledge at the transmitter or receiver. The second part concentrates on spatial multiplexing to increase data rate by simultaneously transmitting multiple data streams without additional bandwidth or power expenditure. Several basic receiver architectures for handling inter-antenna interference, including zero-forcing (ZF), minimum mean square error (MMSE), interference cancellation, etc., are then introduced. The third part of this chapter introduces antenna beamforming techniques to increase signal-tointerference plus noise ratio (SINR) by coherently combining signals with different phase and amplitude at the transmitter or receiver, also known as transmit beamforming or receive beamforming. Another benefit of adopting beamforming is to facilitate multiuser accesses in spatial domain and effectively control multiuser interference. The optimal designs of these beamforming schemes are also presented in this chapter.

\section{Diversity techniques}

Diversity techniques have been widely adopted in modern communications to overcome multipath fading, which allows for enhancing the reliability of signal reception without sacrificing additional transmission power and bandwidth (Rappaport, 2002; Simon \& Alouini, 1999). The basic idea of diversity is that multiple replicas of transmitted signals which carry the same information, but experience independent or small correlated fading, 
are available at the receiver. In fading channels, some samples are severely faded, while others are less attenuated; hence, in statistics, the probability of the signal strength of all samples being simultaneously below a given level becomes small, as compared with the case without applying diversity techniques. Consequently, we can overwhelm the channel fading by imposing an appropriate selection or combination of various samples, so as to dramatically improve the signal quality. Based on signal processing domains to obtain diversity gains, diversity techniques can be classified into time, frequency and space diversity. Here, we focus on space diversity techniques where multiple antennas are deployed at the transmitter or receiver sides. One category of space diversity schemes is to combine multiple signal replicas at the receiver, which is termed as receive diversity. The other category is to use multiple antennas at the transmitter, and this kind of diversity schemes is called transmit diversity (Giannakis et al., 2006).

In this section, we first present various receive diversity schemes, including selection combining, switch combining, equal-gain combining (EGC), and MRC. The well-known Alamouti's transmit diversity scheme using two transmit antennas and one receive antenna is then introduced. The generalized case using two transmit antennas and multiple receive antennas is shown as well. Subsequently, space-time block codes (STBCs) with the number of transmit antennas larger than two (Tarokh et al., 1999) are presented. Finally, a maximum ratio transmission (MRT) scheme is discussed to simultaneously achieve both transmit and receive diversity gains and maximize the output signal-to-noise ratio (SNR) (Lo, 1999).

\subsection{Receive diversity techniques}

In cellular systems, receive diversity techniques have been widely applied at base stations for uplink transmission to improve the signal reception quality. This is mainly because base stations can endure larger implementation size, power consumption, and cost. In general, the performance of the receive diversity not only depends on the number of antennas but also the combining methods utilized at the receiver side. According to the implementation complexity and the extent of channel state information required at the receiver, we will introduce four types of combining schemes, including selection combining, switch combining, EGC, and MRC, in the following.

\subsubsection{Selection combining}

Selection combining is a simple receive diversity combining scheme. Consider a receiver equipped with $n_{R}$ receive antennas. Fig. 1 depicts the block diagram of the selection combining scheme. The antenna branch with the largest instantaneous SNR is selected to receive signals at every symbol period. In practical, since it is difficult to measure the SNR, one can implement the selection combining scheme by accumulating and averaging the received signal power, consisting of both signal and noise power, for all antenna branches, and selecting one branch with the highest output signal power.

\subsubsection{Switch combining}

Fig. 2 shows the switch combining diversity scheme. As its name suggested, the receiver scans all the antenna branches and selects a certain branches with the SNR values higher than a preset threshold to receive signals. When the SNR of the selected antenna is dropped down the given threshold due to channel fading, the receiver starts scanning all branches again and switches to other antenna branches. As compared with the selection diversity 
scheme, the switch diversity scheme exhibits lower performance gain since it does not pick up the branch with the highest instantaneous SNR or received signal power. In spite of this performance loss, it is still very attractive for practical implementation as it does not require to periodically and simultaneously monitor all the antenna branches. Another advantage is that since both the selection and switch diversity schemes do not require any knowledge of channel state information, they are not limited to coherent modulation schemes, but can also be applied for noncoherent modulation schemes.

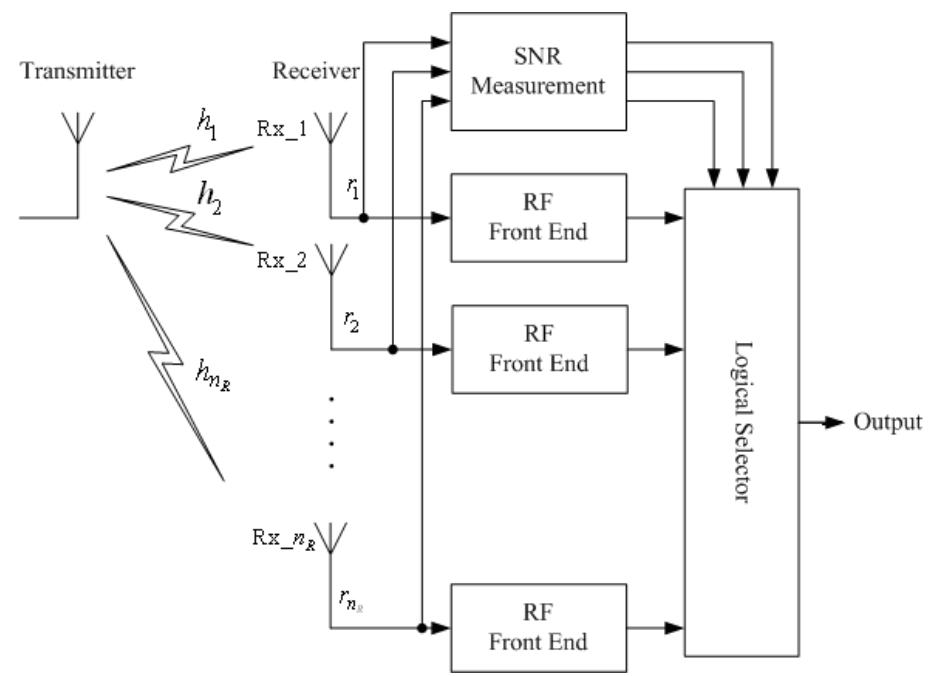

Fig. 1. Block diagram of selection combining scheme

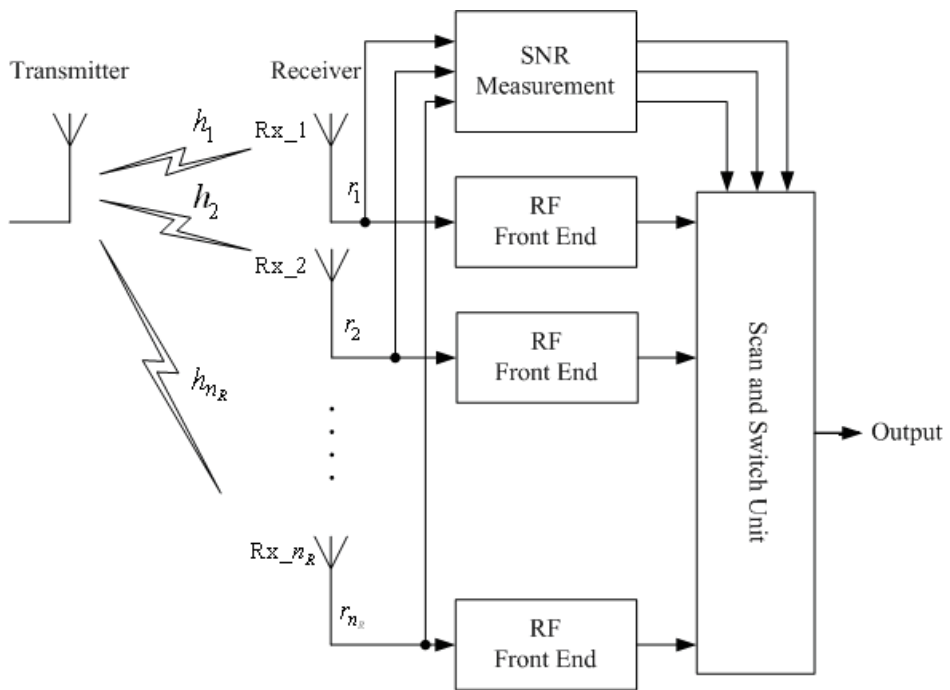

Fig. 2. Block diagram of switch combining scheme 


\subsubsection{Maximum ratio combining}

Fig. 3 shows the block diagram of the MRC scheme. MRC is a linear combining scheme, in which multiple received replicas at the all antenna branches are individually weighted and summed up as an output signal. Since the multiple replicas experience different channel fading gains, the combining scheme can provide diversity gains. In general, there are several ways to determine the weighting factors. Consider a receiver having $n_{R}$ receive antennas, and the received signals can be expressed as a matrix-vector form as follows:

$$
\mathbf{r}=\left[\begin{array}{c}
r_{1} \\
\vdots \\
r_{n_{R}}
\end{array}\right]=\left[\begin{array}{c}
h_{1} \\
\vdots \\
h_{n_{R}}
\end{array}\right] s+\left[\begin{array}{c}
n_{1} \\
\vdots \\
n_{n_{R}}
\end{array}\right]=s \mathbf{h}+\mathbf{n}
$$

where $r_{i}, h_{i}$, and $n_{i}$ are the received signal, channel fading gain, and spatially white noise at the ith receive antenna branch, respectively. After linearly combining the received signals, the output signal is given by

$$
y=\mathbf{w}^{\dagger} \mathbf{r}=\mathbf{w}^{\dagger}(s \mathbf{h}+\mathbf{n})=s \mathbf{w}^{\dagger} \mathbf{h}+\mathbf{w}^{\dagger} \mathbf{n}
$$

where $\mathbf{w}$ represents the weighting factors for all antenna branches, and $(\cdot)^{\dagger}$ is the Hermitian operation. Subsequently, from (2), for a given $\mathbf{h}$, the output SNR is calculated by

$$
\operatorname{SNR}_{o}=\frac{E_{s}\left|\mathbf{w}^{\dagger} \mathbf{h}\right|^{2}}{\sigma_{n}^{2}\|\mathbf{w}\|^{2}}
$$

where $E_{s}$ and $\sigma_{n}^{2}$ are the signal power and the noise power, respectively. According to the Cauchy-Schwarz inequality, we have

$$
\left|\mathbf{w}^{\dagger} \mathbf{h}\right|^{2} \leq\|\mathbf{w}\|^{2}\|\mathbf{h}\|^{2}
$$

Hence, the upper bound for the output SNR is given by

$$
S N R_{o}=\frac{E_{s}\left|\mathbf{w}^{\dagger} \mathbf{h}\right|^{2}}{\sigma_{n}^{2}\|\mathbf{w}\|^{2}} \leq \frac{E_{s}\|\mathbf{h}\|^{2}}{\sigma_{n}^{2}}=\|\mathbf{h}\|^{2} S N R_{i}
$$

where $S N R_{i}=E_{s} / \sigma_{n}^{2}$ is defined as the input SNR. We can further observe that the equality in (5) holds if and only if $\mathbf{w}=\mathbf{h}$, and therefore, the maximum output SNR can be written as

$$
S N R_{o}=\|\mathbf{h}\|^{2} S N R_{i}
$$

The method adopting weighting factors $\mathbf{w}=\mathbf{h}$ is called MRC, as it is capable of maximizing the output SNR with a combining gain of $\|\mathbf{h}\|^{2}$. However, the main drawback of the MRC scheme is that it requires the complete knowledge of channel state information, including both amplitude and phase of $h_{i}$, to coherently combine all the received signals. Hence, it is not suitable for noncoherent modulation schemes. 


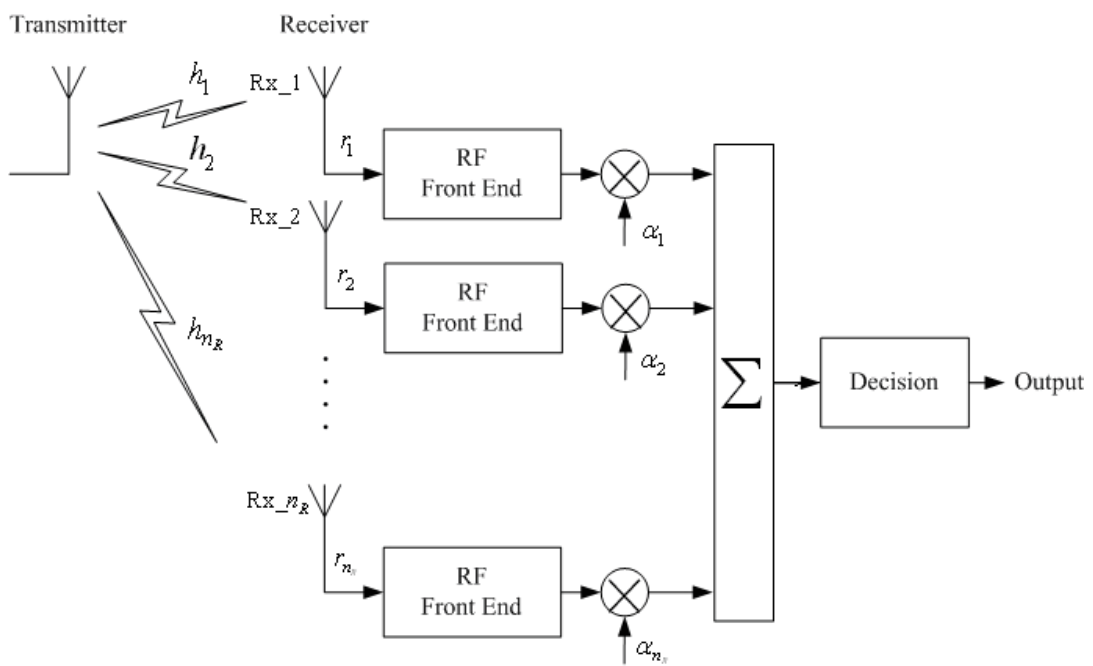

Fig. 3. Block diagram of MRC scheme

\subsubsection{Equal gain combining}

Equal gain combing is a suboptimal combining scheme, as compared with the MRC scheme. Instead of requiring both the amplitude and phase knowledge of channel state information, it simply needs phase information for each individual channels, and set the amplitude of the weighting factor on each individual antenna branch to be unity. Thus, all multiple received signals are combined in a co-phase manner with an equal gain. The performance of the equal gain combining scheme is only slightly worse than that of the MRC scheme, while its implementation cost is significantly less than that of the MRC scheme.

\subsection{Transmit diversity techniques}

Although the receive diversity can provide great benefits for uplink transmission, it is difficult to utilize the receive diversity techniques at mobile terminals for downlink transmission. First, it is hard to place more than two antenna elements in a small-size portable mobile device. Second, multiple chains of radio frequency components will increase power consumption and implementation cost. Since mobile devices are usually battery-limited and cost-oriented, it is impractical and uneconomical for using multiple antennas at the mobile terminals to gain diversity gains at forward links. For these reasons, transmit diversity techniques are deemed as a very attractive alternative. Wittneben (Wittneben, 1993) proposed a delay diversity scheme, where replicas of the same symbol are transmitted through multiple antennas at different time slots to impose an artificial multipath. A maximum likelihood sequence estimator (MLSE) or a MMSE equalizer is subsequently used to obtain spatial diversity gains. Another interesting approach is STC, which can be divided into two categories: space-time trellis codes (STTCs) (Tarokh et al., 1998) and STBCs. In the STTC scheme, encoded symbols are simultaneously transmitted through different antennas and decoded using a maximum likelihood (ML) decoder. This scheme combines the benefits of coding gain and diversity gain, while its complexity grows exponentially with the bandwidth efficiency and achievable diversity order. Therefore, it 
may be not practical or cost-effective for some applications. Alamouti's STC was historically the first STBC to provide two- branch transmit diversity gains for a communication system equipped with two transmit antennas. It has been recognized as a remarkable, but simple, diversity technique, and adopted in a number of next-generation wireless standards, e.g., 3GPP long-term evolution and IEEE 802.16e standards.

In this section, we overview Alamouti's transmit diversity technique. We focus on both encoding and decoding algorithms, along with its performance results. Then, we introduce the generalized STBCs with an arbitrary number of transmit antennas to achieve full diversity gains, which are proposed by Vahid Tarokh (Tarokh et al., 1999) based on orthogonal design theory.

\subsubsection{Alamouti's space-time encoding}

The encoding procedure of Alamouti's Space-time codes for a two-transmit antenna system is depicted in Fig. 4. Assume that data symbols, each of which is mapped from a group of $m$ information bits through an $M$-ary modulation scheme, are going to be transmitted, where $m=\log _{2} M$. Let $\mathbf{C}$ denote the set of constellation points. For each encoding round, the encoder successively takes a pair of two modulated data symbols $x_{1} \in \mathbf{C}$ and $x_{2} \in \mathbf{C}$ to generate two transmit signal sequences of length two, according to the following space-time encoding matrix:

$$
\mathbf{X}=\left[\begin{array}{cc}
x_{1} & -x_{2}^{*} \\
x_{2} & x_{1}^{*}
\end{array}\right]
$$

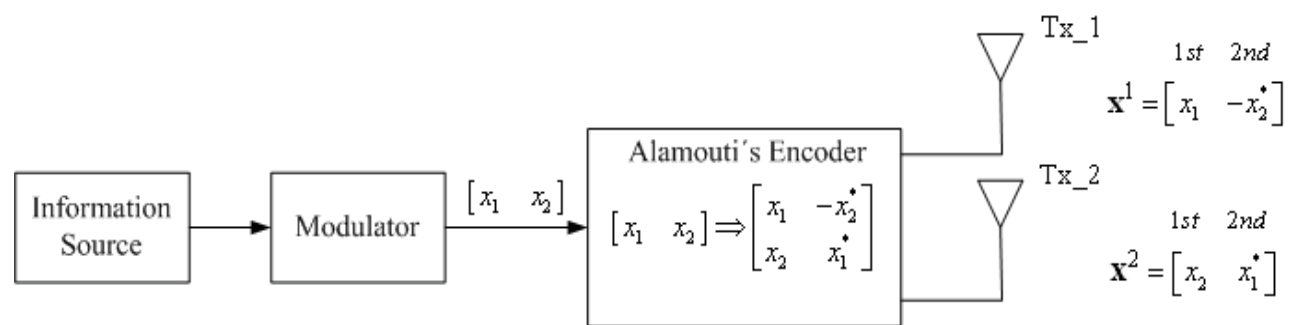

Fig. 4. Block diagram of Alamouti's space-time encoder

The Alamouti's STC is a two-dimensional code, in which the encoder outputs are transmitted within two consecutive time slots over two transmit antennas. During the first time slot, two signals $x_{1}$ and $x_{2}$ are transmitted simultaneously from antenna one and antenna two, respectively. Similarly, in the second time slot, the signal $-x_{2}{ }^{*}$ is transmitted from antenna one and the signal $x_{1}{ }^{*}$ is from antenna two, where $(\cdot)^{*}$ denotes the complex conjugate operation. It is clear that the encoding process is accomplished in both spatial and temporal domains. Let us first denote the transmit sequence from antenna one and two by $\mathbf{x}^{1}$ and $\mathbf{x}^{2}$, respectively, as

$$
\begin{aligned}
& \mathbf{x}^{1}=\left[\begin{array}{ll}
x_{1} & -x_{2}^{*}
\end{array}\right] \\
& \mathbf{x}^{2}=\left[\begin{array}{ll}
x_{2} & x_{1}^{*}
\end{array}\right]
\end{aligned}
$$


We can observe that these two signal sequences possess the orthogonal property with each other. That is, we have

$$
\mathbf{x}^{1}\left(\mathbf{x}^{2}\right)^{\dagger}=x_{1} x_{2}^{*}-x_{2}^{*} x_{1}=0
$$

Where $(\cdot)^{\dagger}$ denotes the Hermitian operation.

In other words, the code matrix, $\mathbf{X}$, satisfies the orthogonal matrix property as follows:

$$
\begin{aligned}
\mathbf{X X}^{\dagger} & =\left[\begin{array}{cc}
\left|x_{1}\right|^{2}+\left|x_{2}\right|^{2} & 0 \\
0 & \left|x_{1}\right|^{2}+\left|x_{2}\right|^{2}
\end{array}\right] \\
& =\left(\left|x_{1}\right|^{2}+\left|x_{2}\right|^{2}\right) \mathbf{I}_{2}
\end{aligned}
$$

where $\mathbf{I}_{2}$ is a $2 \times 2$ identity matrix.

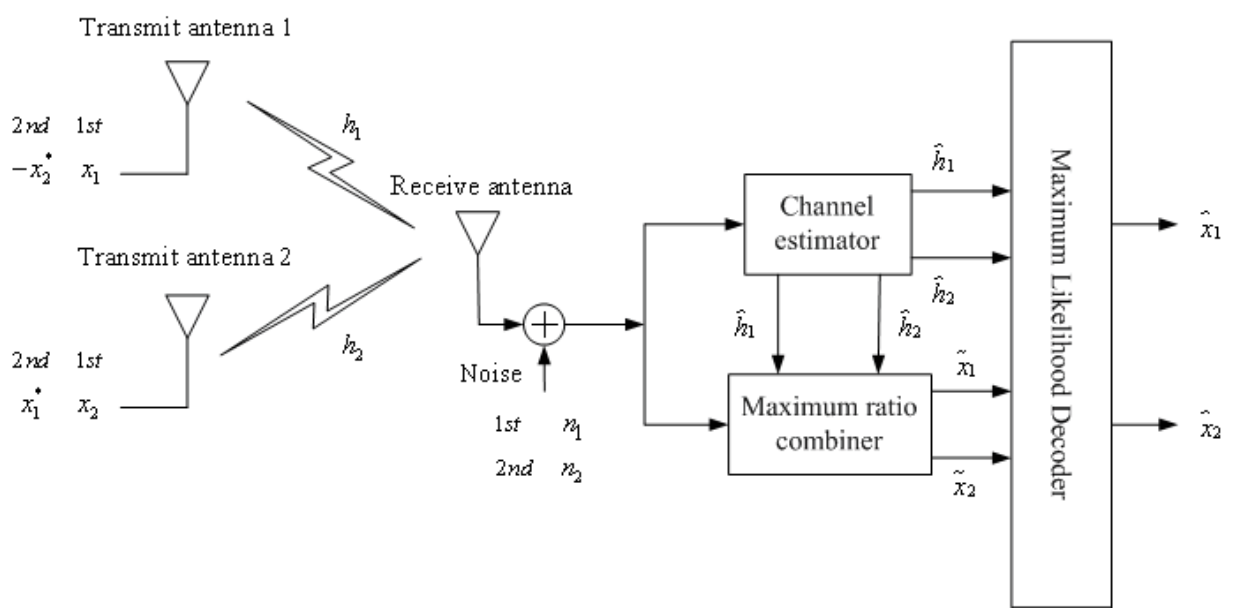

Fig. 5. Block diagram of Alamouti's space-time encoder

Let us assume that there is only one receive antenna deployed at the receiver side. The receiver block diagram for the Alamouti's scheme is shown in Fig. 5. Assume that flat fading channel gains from transmit antenna one and two to the receive antenna at the time slot $t$ are denoted by $h_{1}(t)$ and $h_{2}(t)$, respectively. Under the assumption of quasi-static channels, the channel gains across two consecutive symbol periods remain unchanged, and they can be expressed as follows:

$$
h_{1}(t)=h_{1}(t+T)=h_{1}
$$

and

$$
h_{2}(t)=h_{2}(t+T)=h_{2}
$$

where $h_{i}$, for $i=1$ and 2 , is a complex constant value corresponding to the channel gain from the transmit antenna $i$ to the receive antenna, and $T$ denotes the symbol period. At 
the receive antenna, the received signals across two consecutive symbol periods, which are denoted by $r_{1}$ and $r_{2}$ for time $t$ and $t+T$, are respectively given by

$$
r_{1}=h_{1} x_{1}+h_{2} x_{2}+n_{1}
$$

and

$$
r_{2}=-h_{1} x_{2}^{*}+h_{2} x_{1}^{*}+n_{2}
$$

where $n_{1}$ and $n_{2}$ are independent additive white Gaussian noise with zero mean and variance $\sigma^{2}$. It is noticed here that although we present Alamouti's space-time codes under flat fading channels without concerning the multipath effect, it is straightforward to extend the Alamouti's scheme to the case of multipath channels by using an OFDM technique to transform a frequency selective fading channel into a number of parallel flat fading channels (Ku \& Huang, 2006).

\subsubsection{Maximum likelihood decoding for Alamouti's scheme}

The successful decoding for Alamouti's space-time codes requires the knowledge on channel state information $h_{1}$ and $h_{2}$ at the receiver side. In general, channel estimation can be performed through the use of some pilot signals which are frequently transmitted from the transmit side (Ku \& Huang, 2008; Lin, 2009a, 2009b). Here, we focus on the decoding scheme and assume that channel state information is perfectly estimated and known to the receiver. From the viewpoint of minimum error probability, the decoder intends to choose an optimal pair of constellation points, $\left(\hat{x}_{1}, \hat{x}_{2}\right)$, to maximize the a posteriori probability given by the received signals $r_{1}$ and $r_{2}$. Mathematically, we can express the decoding problem as

$$
\left(\hat{x}_{1}, \hat{x}_{2}\right)=\arg \max _{\left(x_{1}, x_{2}\right) \in \mathbf{C}^{2}} \operatorname{Pr}\left(x_{1}, x_{2} \mid r_{1}, r_{2}\right)
$$

where $\mathbf{C}^{2}$ is the set of all possible candidate symbol pairs $\left(x_{1}, x_{2}\right)$, and $\operatorname{Pr}(\cdot)$ is a probability notation. According to the Bayes' theorem, we can further expand (15) as

$$
\left(\hat{x}_{1}, \hat{x}_{2}\right)=\arg \max _{\left(x_{1}, x_{2}\right) \in \mathbf{C}^{2}} \frac{\operatorname{Pr}\left(r_{1}, r_{2} \mid x_{1}, x_{2}\right) \mathrm{P}\left(x_{1}, x_{2}\right)}{\mathrm{P}\left(r_{1}, r_{2}\right)}
$$

By assuming that all the constellation points in $C^{2}$ occur with equal prior probabilities and the two symbols of each pair are generated independently, all symbol pairs $\left(x_{1}, x_{2}\right)$ are equiprobable. As the decision of the symbol pairs $\left(x_{1}, x_{2}\right)$ is irrelevant to the probability of received signals $r_{1}$ and $r_{2}$, we can rewrite (16) as

$$
\left(\hat{x}_{1}, \hat{x}_{2}\right)=\arg \max _{\left(x_{1}, x_{2}\right) \in \mathrm{C}^{2}} \operatorname{Pr}\left(r_{1}, r_{2} \mid x_{1}, x_{2}\right)
$$

Furthermore, since the noise $n_{1}$ and $n_{2}$ at time $t$ and time $t+T$, respectively, are assumed to be mutually independent, we can alternatively express (17) as

$$
\left(\hat{x}_{1}, \hat{x}_{2}\right)=\arg \max _{\left(x_{1}, x_{2}\right) \in \mathrm{C}^{2}} \operatorname{Pr}\left(r_{1} \mid x_{1}, x_{2}\right) \operatorname{Pr}\left(r_{2} \mid x_{1}, x_{2}\right)
$$


Recall from (13) and (14) that $r_{1}$ and $r_{2}$ are two independent Gaussian random variables with distributions $r_{1} \sim N\left(h_{1} x_{1}+h_{2} x_{2}, \sigma^{2}\right)$ and $r_{2} \sim N\left(-h_{1} x_{2}^{*}+h_{2} x_{1}^{*}, \sigma^{2}\right)$. Substituting this into (18), we then obtain a ML decoding criterion:

$$
\begin{aligned}
\left(\hat{x}_{1}, \hat{x}_{2}\right) & =\arg \min _{\left(x_{1}, x_{2}\right) \in \mathrm{C}^{2}}\left|r_{1}-h_{1} x_{1}-h_{2} x_{2}\right|^{2}+\left|r_{2}+h_{1} x_{2}^{*}-h_{2} x_{1}^{*}\right|^{2} \\
& =\arg \min _{\left(x_{1}, x_{2}\right) \in \mathrm{C}^{2}} d^{2}\left(r_{1}, h_{1} x_{1}+h_{2} x_{2}\right)+d^{2}\left(r_{2},-h_{1} x_{2}^{*}+h_{2} x_{1}^{*}\right)
\end{aligned}
$$

where $d^{2}\left(s_{1}, s_{2}\right)$ denotes the Euclidean distance between $s_{1}$ and $s_{2}$. The ML decoder is, therefore, equivalent to choosing a pair of data symbols $\left(\hat{x}_{1}, \hat{x}_{2}\right)$ to minimize the distance metric, as indicated in (19). By replacing (13) and (14) into (19), the ML decoding criterion can be further rewritten as a meaningful expression as follows:

$$
\left(\hat{x}_{1}, \hat{x}_{2}\right)=\arg \min _{\left(x_{1}, x_{2}\right) \in \mathrm{C}^{2}}\left(\left|h_{1}\right|^{2}+\left|h_{2}\right|^{2}-1\right)\left(\left|x_{1}\right|^{2}+\left|x_{2}\right|^{2}\right)+d^{2}\left(\tilde{x}_{1}, x_{1}\right)+d^{2}\left(\tilde{x}_{2}, x_{2}\right)
$$

where $\tilde{x}_{1}$ and $\tilde{x}_{2}$ are two decision statistics obtained by combining the received signals $r_{1}$ and $r_{2}$ with channel state information $h_{1}$ and $h_{2}$, given by

$$
\begin{aligned}
& \tilde{x}_{1}=h_{1}^{*} r_{1}+h_{2} r_{2}^{*} \\
& \tilde{x}_{2}=h_{2}^{*} r_{1}+h_{1} r_{2}^{*}
\end{aligned}
$$

By taking $r_{1}$ and $r_{2}$ from equation (13) and (14), respectively, into (21), the decision statistics is given by

$$
\begin{aligned}
& \tilde{x}_{1}=\left(\left|h_{1}\right|^{2}+\left|h_{2}\right|^{2}\right) x_{1}+h_{1}^{*} n_{1}+h_{2} n_{2}^{*} \\
& \tilde{x}_{2}=\left(\left|h_{1}\right|^{2}+\left|h_{2}\right|^{2}\right) x_{2}-h_{1} n_{2}^{*}+h_{2}^{*} n_{1}
\end{aligned}
$$

It is observed that for a given channel realization $h_{1}$ and $h_{2}$, the decision statistics $\tilde{x}_{i}$ in (22) is only a function of $x_{i}$, for $i=1,2$. Consequently, the ML decoding criterion in (20) can be divided into two independent decoding criteria for $x_{1}$ and $x_{2}$; that is, we have

$$
\hat{x}_{1}=\arg \min _{x_{1} \in \mathbf{C}}\left(\left|h_{1}\right|^{2}+\left|h_{2}\right|^{2}-1\right)\left|x_{1}\right|^{2}+d^{2}\left(\tilde{x}_{1}, x_{1}\right)
$$

and

$$
\hat{x}_{2}=\arg \min _{x_{2} \in \mathbf{C}}\left(\left|h_{1}\right|^{2}+\left|h_{2}\right|^{2}-1\right)\left|x_{2}\right|^{2}+d^{2}\left(\tilde{x}_{2}, x_{2}\right)
$$

Particularly, if a constant envelope modulation scheme such as $M$-phase-shift-keying ( $M$ PSK) is adopted, the term $\left(\left|h_{1}\right|^{2}+\left|h_{2}\right|^{2}-1\right)\left|x_{i}\right|^{2}$, for $i=1,2$, remains unchanged for all possible signal points with a fixed channel fading coefficients $h_{1}$ and $h_{2}$. Under this circumstance, the decision rules of (23) and (24) can be further simplified as

$$
\hat{x}_{1}=\arg \min _{x_{1} \in \mathbf{C}} d^{2}\left(\tilde{x}_{1}, x_{1}\right) ; \quad \hat{x}_{2}=\arg \min _{x_{2} \in \mathbf{C}} d^{2}\left(\tilde{x}_{2}, x_{2}\right)
$$


From (25), for the case of constant envelope modulation, the decoding algorithm is just a linear decoder with extremely low complexity to achieve diversity gains. On the other hand, when non-constant envelope modulation, e.g., quadrature-amplitude-modulation (QAM) is adoped, the term $\left(\left|h_{1}\right|^{2}+\left|h_{2}\right|^{2}-1\right)\left|x_{i}\right|^{2}$, for $i=1,2$, may become different for various constellation points and cannot be excluded from the decoding metric. Therefore, we should follow the decoding rules as shown in (23) and (24) to achieve the ML decoding.

\subsubsection{Alamouti's scheme with multiple receive antennas}

We now extend the Alamouti's scheme to an MIMO communication system with $n_{R}$ multiple receive antennas. Let us denote $r_{1}^{j}$ and $r_{2}^{j}$ as the received signals at the $j$ th receive antenna at the time slot $t$ and $t+T$, respectively. According to (13) and (14), it follows

$$
\begin{aligned}
& r_{1}^{j}=h_{j, 1} x_{1}+h_{j, 2} x_{2}+n_{1}^{j} \\
& r_{2}^{j}=-h_{j, 1} x_{2}^{*}+h_{j, 2} x_{1}^{*}+n_{2}^{j}
\end{aligned}
$$

where $h_{j, i}$, for $i=1,2$ and $j=1, \cdots, n_{R}$, is the channel fading gain from the transmit antenna $i$ to the receive antenna $j$, and $n_{1}^{j}$ and $n_{2}^{j}$ are assumed to be spatially and temporally white Gaussian noises for the receive antenna $j$ at time $t$ and $t+T$, respectively. Similar to the derivation in the case of single receive antenna, the ML decoding criterion with multiple receive antennas now can be formulated as below:

$$
\begin{aligned}
\left(\hat{x}_{1}, \hat{x}_{2}\right) & =\arg \min _{\left(x_{1}, x_{2}\right) \in \mathbf{C}^{2}} \sum_{j=1}^{n_{R}}\left|r_{1}^{j}-h_{j, 1} x_{1}-h_{j, 2} x_{2}\right|^{2}+\left|r_{2}^{j}+h_{j, 1} x_{2}^{*}-h_{j, 2} x_{1}^{*}\right|^{2} \\
& =\arg \min _{\left(x_{1}, x_{2}\right) \in \mathbf{C}^{2}} \sum_{j=1}^{n_{R}} d^{2}\left(r_{1}^{j}, h_{j, 1} x_{1}+h_{j, 2} x_{2}\right)+d^{2}\left(r_{2}^{j},-h_{j, 1} x_{2}^{*}+h_{j, 2} x_{1}^{*}\right)
\end{aligned}
$$

We then define two decision statistics by combining the received signals at each receive antenna with the corresponding channel link gains, as follows:

$$
\begin{aligned}
& \tilde{x}_{1}^{j}=h_{j, 1}^{*} r_{1}^{j}+h_{j, 2}\left(r_{2}^{j}\right)^{*} \\
& \tilde{x}_{22}^{j}=h_{j, 2}^{*} r_{1}^{j}-h_{j, 1}\left(r_{2}^{j}\right)^{*}
\end{aligned}
$$

Note that by replacing $r_{1}^{j}$ and $r_{2}^{j}$, given in (26), into (28), the decision statistics can be explicitly written as

$$
\begin{aligned}
& \tilde{x}_{1}^{j}=\left(\left|h_{j, 1}\right|^{2}+\left|h_{j, 2}\right|^{2}\right) x_{1}+h_{j, 1}^{*} n_{1}^{j}+h_{j, 2}\left(n_{2}^{j}\right)^{*} \\
& \tilde{x}_{2}^{j}=\left(\left|h_{j, 1}\right|^{2}+\left|h_{j, 2}\right|^{2}\right) x_{2}-h_{j, 1}\left(n_{2}^{j}\right)^{*}+h_{j, 2}^{*} n_{1}^{j}
\end{aligned}
$$

where $G_{e f f}=\left|h_{j, 1}\right|^{2}+\left|h_{j, 2}\right|^{2}$ is the effective channel fading gain, and it is shown that the Alamouti's STC scheme can therefore extract a diversity order of two at each receiving branch, even in the absence of channel state information at the transmitter side. Following 
the derivation in (19) and (20), the ML decoding rules, under the case of $n_{R}$ receive antennas, for the two data symbols $x_{1}$ and $x_{2}$ can be represented by

$$
\hat{x}_{1}=\arg \min _{x_{1} \in \mathrm{C}} \sum_{j=1}^{n_{R}}\left(\left|h_{j, 1}\right|^{2}+\left|h_{j, 2}\right|^{2}-1\right)\left|x_{1}\right|^{2}+d^{2}\left(\tilde{x}_{1}^{j}, x_{1}\right)
$$

and

$$
\hat{x}_{2}=\arg \min _{x_{2} \in \mathbf{C}} \sum_{j=1}^{n_{R}}\left(\left|h_{j, 1}\right|^{2}+\left|h_{j, 2}\right|^{2}-1\right)\left|x_{2}\right|^{2}+d^{2}\left(\tilde{x}_{2}^{j}, x_{2}\right)
$$

In particular, for constant envelope modulation schemes whose constellation points possess equal energy, the ML decoding can be reduced to finding a data symbol $\hat{x}_{i}$, for $i=1,2$, to minimize the summation of Euclidean distance $d^{2}\left(\tilde{x}_{1}^{j}, x_{1}\right)$ over all receive antennas, in the following:

$$
\hat{x}_{1}=\arg \min _{x_{1} \in \mathbf{C}} \sum_{j=1}^{n_{R}} d^{2}\left(\tilde{x}_{1}^{j}, x_{1}\right)
$$

and

$$
\hat{x}_{2}=\arg \min _{x_{2} \in \mathbf{C}} \sum_{j=1}^{n_{R}} d^{2}\left(\tilde{x}_{2}^{j}, x_{2}\right)
$$

\subsubsection{BER performance of Alamouti's scheme}

The bit error rate (BER) performance of the Alamouti's transmit diversity scheme is simulated and compared with the MRC receive diversity scheme in the following. At the beginning, it is assumed that a flat fading channel is used, and the fading gains from each transmit antenna to each receive antenna are mutually independent. Furthermore, we assume that the total transmission power from the two transmit antennas for the Alamouti's scheme is the same as that for the MRC receive diversity scheme.

Fig. 6 compares the BER performance between the Alamouti's with one or two receive antennas and the MRC receive diversity with two or four receive antennas. The Alamouti's scheme with two transmit antennas and one receive antenna has the same diversity order as the MRC receive diversity scheme with two branches. In other words, the slopes of these two BER performance curves are identical. However, the Alamouti's scheme has $3 \mathrm{~dB}$ loss in terms of $E_{b} / N_{0}$. This is due to the fact that for fair comparisons, the total transmission power is fixed and the energy radiated from each transmit antenna in the Alamouti's scheme is a half of that from a single transmit antenna in the MRC receive diversity scheme. Similarly, the Alamouti's scheme with two receive antennas can introduce the same diversity order as the MRC receiver diversity scheme with four branches, while there is still $3 \mathrm{~dB}$ loss in BER performance. In general, the Alamouti's scheme with two transmit antennas and $n_{R}$ receive antennas can provide a diversity order of $2 \times n_{R}$, which is the same as the case that the MRC scheme uses $2 n_{R}$ receive antennas.

In Fig. 7, it is shown that the BER performance of the Alamouti's scheme with quadrature phase-shift keying (QPSK) modulation over flat fading channels. It is obvious that the more number of receive antennas it uses, the higher diversity order it can achieves. 


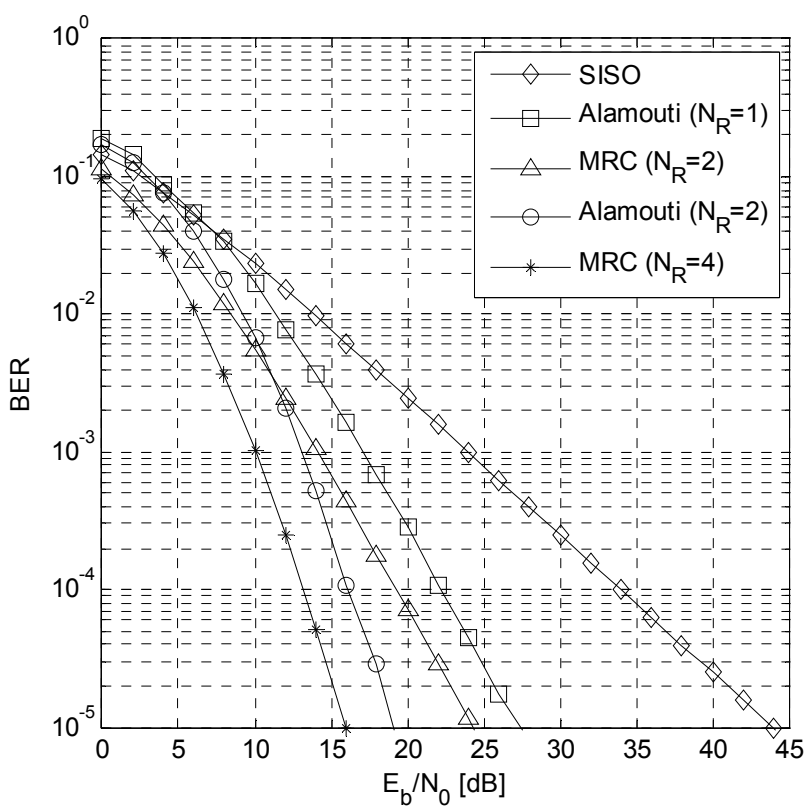

Fig. 6. Comparison of BER performance between Alamouti's and MRC schemes with binary phase-shift keying (BPSK) modulation

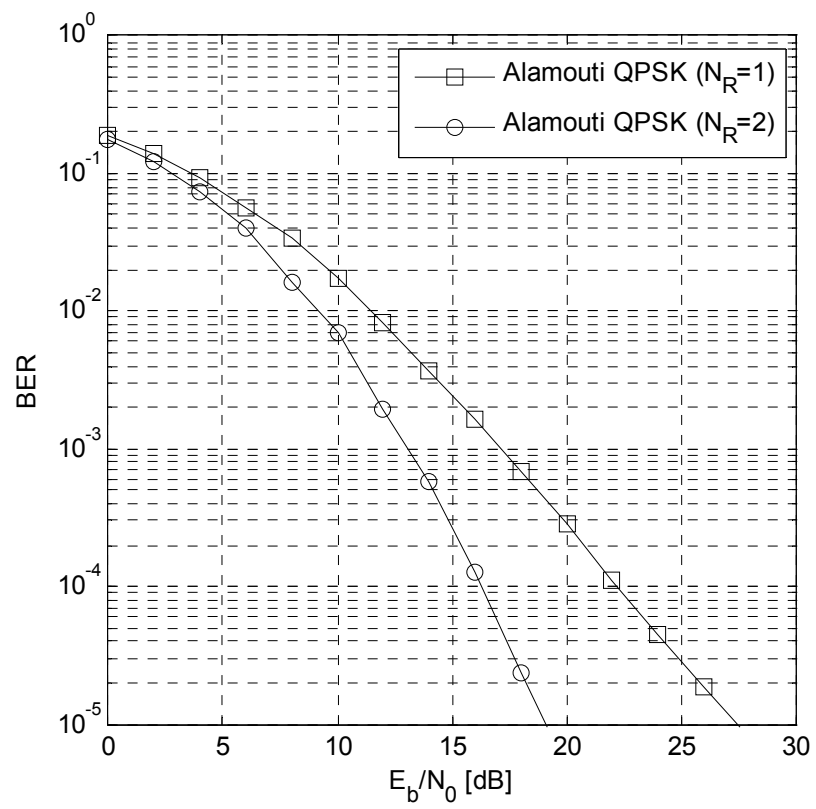

Fig. 7. BER performance of Alamouti's scheme using QPSK modulation 


\subsubsection{Generalized space-time block codes}

As we discussed, the Alamouti's scheme shows a very elegant way to achieve full diversity gains, i.e., a diversity order of two, with a low-complexity linear decoding algorithm by utilizing two transmit antennas. The key feature of the Alamouti's scheme is the orthogonal property of the encoding matrix in (1), i.e., the sequences generated by the two transmit antennas are independent of each other. In (Tarokh et al., 1999), Tarokh generalizes this idea to any arbitrary number of transmit antennas by applying the orthogonal design theory, and proposes a series of STBCs which can fulfill transmit diversity specified by the number of transmit antennas $n_{T}$. Meanwhile, these STBCs also enable a very simple maximumlikelihood decoding algorithm, based only on a linear processing of the received signals at different time slots.

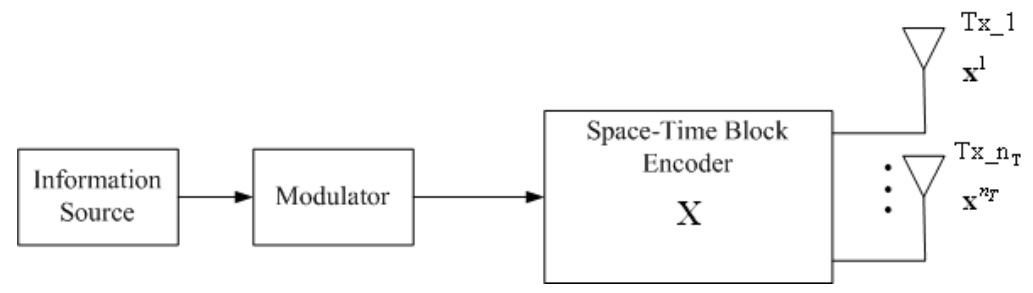

Fig. 8. Encoder structure of STBCs

The encoder structure for generalized STBCs is presented in Fig. 8. In general, a STBC can be defined via an $n_{T} \times p$ transmission matrix $\mathbf{X}$, where $n_{T}$ represents the number of transmit antennas and $p$ is the time duration for transmitting each block of space-time coded symbols. Consider a $M$-ary modulation scheme, where we define $m=\log _{2} M$ as the number of information bits required for each constellation point mapping. At each encoding operation, a block of $k m$ information bits are mapped onto $k$ modulated data symbols $x_{i}$, for $i=1, \ldots, k$. Subsequently, these $k$ modulated symbols are encoded by the $n_{T} \times p$ spacetime encoder $\mathbf{X}$ to generate $n_{T}$ parallel signal sequences of length $p$ which are to be transmitted over $n_{T}$ transmit antennas simultaneously within $p$ time slots. The code rate of a STBC is defined as the ratio of the number of symbols taken by the space-time encoder as its input to the number of space-time coded symbols transmitted from each antenna. Since $p$ time slots are required for transmitting $k$ information-bearing data symbols, the code rate is given by

$$
R_{c} \triangleq \frac{k}{p}
$$

Therefore, the spectral efficiency for the STBC is calculated by

$$
\eta \triangleq \frac{r_{b}}{B}=\frac{\left(r_{s} m\right) R_{c}}{r_{s}}=\frac{k m}{p}(\mathrm{bits} / \mathrm{sec} / \mathrm{Hz})
$$

where $r_{b}$ and $r_{s}$ are the bit and symbol rate of a space-time coded symbol, respectively, and $B$ represents the total bandwidth. For simplicity of notations, we usually denote a STBC with $n_{T}$ transmit antennas as $\mathbf{X}_{n_{T}}$. Based on the orthogonal designs in (Tarokh et al., 1999), to obtain full diversity gains, i.e., diversity order is equal to $n_{T}$, the space-time encoding matrix should preserve the orthogonal structure; that is, we have 


$$
\mathbf{X}_{n_{T}} \cdot \mathbf{X}_{n_{T}}^{\dagger}=c\left(\left|x_{1}\right|^{2}+\left|x_{2}\right|^{2}+\cdots+\left|x_{k}\right|^{2}\right) \mathbf{I}_{n_{T}}
$$

where $c$ is constant, $(\cdot)^{\dagger}$ takes the Hermitian operation, and $\mathbf{I}_{n_{T}}$ is an $n_{T} \times n_{T}$ identity matrix, the entries in $\mathbf{X}_{n_{T}}$ take the values of modulated symbols $x_{i}$, their conjugate $x_{i}^{*}$, or their combination. The orthogonal structure allows the receiver to decouple the signals transmitted from different antennas by using a simple linear decoder derived based on the ML decoding metric. Tarokh et al. (Tarokh et al., 1999) discovered that the code rate of a STBC with full diversity must be less than or equal to one, i.e., $R_{c} \leq 1$. In other words, the STBCs cannot be used to increase bandwidth efficiency, but provide diversity gains. It is noted that the full code rate, $R_{c}=1$, requires no additional bandwidth expansion, while the code rate $R_{c} \leq 1$ requires a bandwidth expansion by a factor of $1 / R_{c}$.

Based on modulation types, STBCs can be classified into two categories: real signaling or complex signaling. For a special case of $p=n_{T}$, it is evident from (Tarokh et al., 1999) that for an arbitrary real constellation signaling, e.g., $M$-amplitude shift keying ( $M$-ASK), STBCs with an $n_{T} \times n_{T}$ square encoding matrices $\mathbf{X}_{n_{T}}$ exist if and only if the number of transmit antennas $n_{T}$ is equal to two, four, or eight. Moreover, these code matrices can not only achieve the full code rate $R_{c}=1$ but also provide the full diversity gains with a diversity order of $n_{T}$. However, it is desirable to have code matrices with the full diversity gains and the full code rate for any number of transmit antennas. It has been proved that for $n_{T}$ transmit antennas, the minimum required value for the transmission periods $p$ to achieve the full diversity $n_{T}$ and the full code rate $R_{c}=1$ must satisfy the following condition:

$$
\min _{\left\{(c, d) \mid 0 \leq c, 0 \leq d \leq 4, \text { and } 8 c+2^{d} \geq n_{T}\right\}} 2^{4 c+d}
$$

\begin{tabular}{||l|l|l}
\hline$n_{T}$ & $p$ & $\mathbf{X}_{n_{T}}$ \\
\hline 2 & 2 & $\mathbf{X}_{2}=\left[\begin{array}{cc}x_{1} & -x_{2} \\
x_{2} & x_{1}\end{array}\right]$ \\
\hline 4 & \multirow{3}{*}{4} & $\mathbf{X}_{4}=\left[\begin{array}{cccc}x_{1} & -x_{2} & -x_{3} & -x_{4} \\
x_{2} & x_{1} & x_{4} & -x_{3} \\
x_{3} & -x_{4} & x_{1} & x_{2} \\
x_{4} & x_{3} & x_{2} & x_{1}\end{array}\right]$ \\
\hline 8 & \multirow{3}{*}{8} & $\mathbf{X}_{8}=\left[\begin{array}{ccccccccc||}x_{1} & -x_{2} & -x_{3} & -x_{4} & -x_{5} & -x_{6} & -x_{7} & -x_{8} \\
x_{2} & x_{1} & -x_{4} & x_{3} & -x_{6} & x_{5} & x_{8} & -x_{7} \\
x_{3} & x_{4} & x_{1} & -x_{2} & -x_{7} & -x_{8} & x_{5} & x_{6} \\
x_{4} & -x_{3} & x_{2} & x_{1} & -x_{8} & x_{7} & -x_{6} & x_{5} \\
x_{5} & x_{6} & x_{7} & x_{8} & x_{1} & -x_{2} & -x_{3} & -x_{4} \\
x_{6} & -x_{5} & x_{8} & -x_{7} & x_{2} & x_{1} & x_{4} & -x_{3} \\
x_{7} & -x_{8} & -x_{5} & x_{6} & x_{3} & -x_{4} & x_{1} & x_{2} \\
x_{8} & x_{7} & -x_{6} & -x_{5} & x_{4} & x_{3} & -x_{2} & x_{1}\end{array}\right]$ \\
\hline
\end{tabular}

Table 1. Square code matrices with full diversity gains and full code rate for $n_{T}=2,4,8$ 
Accordingly, the minimum values of $p$ for a specific value of $n_{T} \leq 8$, and the associated STBC matrices $\mathbf{X}_{n_{T}}$ for real signaling are provided as follows, where the square transmission matrices $\mathbf{X}_{2}, \mathbf{X}_{4}$, and $\mathbf{X}_{8}$ are listed in Table 1 , and the non-square transmission matrices $\mathbf{X}_{3}, \mathbf{X}_{5}, \mathbf{X}_{6}$ and $\mathbf{X}_{7}$ are listed in Table 2 .

\begin{tabular}{|c|c|c|c|c|c|c|c|c|c|c|}
\hline$n_{T}$ & $p$ & \multicolumn{9}{|c|}{$\overline{\bar{z}} \mathbf{X}_{n_{T}}$} \\
\hline 3 & 4 & $\mathbf{X}_{3}=$ & {$\left[\begin{array}{l}x_{1} \\
x_{2} \\
x_{3}\end{array}\right.$} & $\begin{array}{c}-x_{2} \\
x_{1} \\
-x_{4}\end{array}$ & $\begin{array}{c}-x_{3} \\
x_{4} \\
x_{1}\end{array}$ & $\left.\begin{array}{c}-x_{4} \\
-x_{3} \\
x_{2}\end{array}\right]$ & & & & \\
\hline 5 & 8 & $\mathbf{X}_{5}=$ & {$\left[\begin{array}{l}x_{1} \\
x_{2} \\
x_{3} \\
x_{4} \\
x_{5}\end{array}\right.$} & $\begin{array}{c}-x_{2} \\
x_{1} \\
x_{4} \\
-x_{3} \\
x_{6}\end{array}$ & $\begin{array}{c}-x_{3} \\
-x_{4} \\
x_{1} \\
x_{2} \\
x_{7}\end{array}$ & $\begin{array}{c}-x_{4} \\
x_{3} \\
-x_{2} \\
x_{1} \\
x_{8}\end{array}$ & $\begin{array}{c}-x_{5} \\
-x_{6} \\
-x_{7} \\
-x_{8} \\
x_{1}\end{array}$ & $\begin{array}{c}-x_{6} \\
x_{5} \\
-x_{8} \\
x_{7} \\
-x_{2}\end{array}$ & $\begin{array}{r}-x_{7} \\
x_{8} \\
x_{5} \\
-x_{6} \\
-x_{3}\end{array}$ & $\begin{array}{c}-x_{8} \\
-x_{7} \\
x_{6} \\
x_{5} \\
-x_{4}\end{array}$ \\
\hline 6 & 8 & $X_{6}=$ & {$\left[\begin{array}{l}x_{1} \\
x_{2} \\
x_{3} \\
x_{4} \\
x_{5} \\
x_{6}\end{array}\right.$} & $\begin{array}{c}-x_{2} \\
x_{1} \\
x_{4} \\
-x_{3} \\
x_{6} \\
-x_{5}\end{array}$ & $\begin{array}{c}-x_{3} \\
-x_{4} \\
x_{1} \\
x_{2} \\
x_{7} \\
x_{8}\end{array}$ & $\begin{array}{c}-x_{4} \\
x_{3} \\
-x_{2} \\
x_{1} \\
x_{8} \\
-x_{7}\end{array}$ & $\begin{array}{c}-x_{5} \\
-x_{6} \\
-x_{7} \\
-x_{8} \\
x_{1} \\
x_{2}\end{array}$ & $\begin{array}{c}-x_{6} \\
x_{5} \\
-x_{8} \\
x_{7} \\
-x_{2} \\
x_{1}\end{array}$ & $\begin{array}{c}-x_{7} \\
x_{8} \\
x_{5} \\
-x_{6} \\
-x_{3} \\
x_{4}\end{array}$ & $\begin{array}{c}-x_{8} \\
-x_{7} \\
x_{6} \\
x_{5} \\
-x_{4} \\
-x_{3}\end{array}$ \\
\hline 7 & 8 & $X_{7}=$ & {$\left[\begin{array}{l}x_{1} \\
x_{2} \\
x_{3} \\
x_{4} \\
x_{5} \\
x_{6} \\
x_{7}\end{array}\right.$} & $\begin{array}{c}-x_{2} \\
x_{1} \\
x_{4} \\
-x_{3} \\
x_{6} \\
-x_{5} \\
-x_{8}\end{array}$ & $\begin{array}{c}-x_{3} \\
-x_{4} \\
x_{1} \\
x_{2} \\
x_{7} \\
x_{8} \\
-x_{5}\end{array}$ & $\begin{array}{c}-x_{4} \\
-x_{3} \\
-x_{2} \\
x_{1} \\
x_{8} \\
-x_{7} \\
x_{6}\end{array}$ & $\begin{array}{c}-x_{5} \\
-x_{6} \\
-x_{7} \\
-x_{8} \\
x_{1} \\
x_{2} \\
x_{3}\end{array}$ & $\begin{array}{c}-x_{6} \\
x_{5} \\
-x_{8} \\
x_{7} \\
-x_{2} \\
x_{1} \\
-x_{4}\end{array}$ & $\begin{array}{c}-x_{7} \\
x_{8} \\
x_{5} \\
-x_{6} \\
-x_{3} \\
x_{4} \\
x_{1}\end{array}$ & $\begin{array}{c}-x_{8} \\
-x_{7} \\
x_{6} \\
x_{5} \\
-x_{4} \\
-x_{3} \\
x_{2}\end{array}$ \\
\hline
\end{tabular}

Table 2. Non-square code matrices with full diversity gains and full code rate for $n_{T}=3,5,6,7$

The other type of STBCs belongs to complex constellation signaling, and just as the case for the real constellation signaling, these complex STBCs also abide by the orthogonal design constraint in (36). In particular, Alamouti's scheme can be regarded as a complex STBC for two transmit antennas; that is, the code matrix can be expressed as

$$
\mathbf{X}_{2}^{C}=\left[\begin{array}{cc}
x_{1} & -x_{2}^{*} \\
x_{2} & x_{1}^{*}
\end{array}\right]
$$

where we use $\mathbf{X}_{n_{T}}^{C}$ to denote a complex STBC for $n_{T}$ transmit antennas in order to discriminate between real and complex matrices. It is noted that the Alamouti's scheme can provide a diversity order of two and the full code rate. As compared with those real STBCs, it is much more desirable to invent complex STBCs since complex constellation schemes 
usually exhibit higher bandwidth efficiency. In addition, one might wonder if there exist other complex STBC matrices for $n_{T}>2$. Unfortunately, it has been shown that Alamouti's scheme is the only complex STBC with an $n_{T} \times n_{T}$ square code matrix to simultaneously achieve the full diversity gains and the full code rate. For the case of $n_{T}>2$, we might intend to construct complex orthogonal matrices $\mathbf{X}_{n_{T}}^{C}$ that can achieve full diversity gains, but with high code rate $R_{c}$ and minimum decoding latency $p$. We summarize below that for any number of transmit antennas, there exist complex STBCs with code rate of $R_{c}=1 / 2$. For example, for the cases of three or four transmit antennas, the code matrices are given by

$$
\begin{aligned}
\mathbf{X}_{3}^{C} & =\left[\begin{array}{cccccccc}
x_{1} & -x_{2} & -x_{3} & -x_{4} & x_{1}^{*} & -x_{2}^{*} & -x_{3}^{*} & -x_{4}^{*} \\
x_{2} & x_{1} & x_{4} & -x_{3} & x_{2}^{*} & x_{1}^{*} & x_{4}^{*} & -x_{3}^{*} \\
x_{3} & -x_{4} & x_{1} & x_{2} & x_{3}^{*} & -x_{4}^{*} & x_{1}^{*} & x_{2}^{*}
\end{array}\right], R_{c}=1 / 2 \\
\mathbf{X}_{4}^{C} & =\left[\begin{array}{cccccccc}
x_{1} & -x_{2} & -x_{3} & -x_{4} & x_{1}^{*} & -x_{2}^{*} & -x_{3}^{*} & -x_{4}^{*} \\
x_{2} & x_{1} & x_{4} & -x_{3} & x_{2}^{*} & x_{1}^{*} & x_{4}^{*} & -x_{3}^{*} \\
x_{3} & -x_{4} & x_{1} & x_{2} & x_{3}^{*} & -x_{4}^{*} & x_{1}^{*} & x_{2}^{*} \\
x_{4} & x_{3} & -x_{2} & x_{1} & x_{4}^{*} & x_{3}^{*} & -x_{2}^{*} & x_{1}^{*}
\end{array}\right], R_{c}=1 / 2
\end{aligned}
$$

Still, for the case of $n_{T}>2$, we can acquire other higher code-rate complex code matrices at the expense of complicated linear encoding and decoding processing. For example, the following two matrices $\boldsymbol{X}_{3}^{C}$ and $\boldsymbol{X}_{4}^{C}$ are STBCs with a code rate of $R_{c}=3 / 4$ :

$$
\left.\begin{array}{rl}
\mathbf{X}_{3}^{C, 3 / 4} & =\left[\begin{array}{cccc}
x_{1} & -x_{2}^{*} & \frac{x_{3}^{*}}{\sqrt{2}} & \frac{x_{3}^{*}}{\sqrt{2}} \\
x_{2} & x_{1}^{*} & \frac{x_{3}^{*}}{\sqrt{2}} & \frac{-x_{3}^{*}}{\sqrt{2}} \\
\frac{x_{3}}{\sqrt{2}} & \frac{x_{3}}{\sqrt{2}} & \frac{\left(-x_{1}-x_{1}^{*}+x_{2}-x_{2}^{*}\right)}{2} & \frac{\left(x_{2}+x_{2}^{*}+x_{1}-x_{1}^{*}\right)}{2}
\end{array}\right], R_{c}=3 / 4 \\
\mathbf{X}_{4}^{C, 3 / 4} & =\left[\begin{array}{cccc}
x_{1} & -x_{2} & \frac{x_{3}^{*}}{\sqrt{2}} & \frac{x_{3}^{*}}{\sqrt{2}} \\
\frac{x_{2}}{x_{3}} & \frac{x_{1}}{\sqrt{2}} & \frac{x_{3}^{*}}{\sqrt{2}} & \frac{\left(-x_{1}-x_{1}^{*}+x_{2}-x_{2}^{*}\right)}{2} \\
\frac{x_{3}}{\sqrt{2}} & \frac{-x_{3}}{\sqrt{2}} & \frac{\left(-x_{2}-x_{2}^{*}+x_{1}-x_{1}-x_{1}^{*}\right)}{2} & \frac{-\left(x_{1}+x_{1}^{*}+x_{2}-x_{2}^{*}\right)}{2}
\end{array}\right], R_{c}=3 / 4
\end{array}\right]
$$

\subsection{Maximum ratio transmission techniques}

The multipath fading is considered as a detrimental effect to degrade the performance of wireless communications. The most common and simplest way to mitigate the multipath 
fading is to adopt antenna diversity techniques. As we introduced in the previous section, with multiple receive antennas, the MRC scheme can attain receive diversity gains and maximize the output SNR, while with multiple transmit antennas, STC schemes are proposed to obtain the MRC-like transmit diversity gains. For example, Alamouti's STC can obtain a diversity order of two by encoding a pair of two symbols to transmit over two transmit antennas and two contiguous time slots. However, these transmit diversity techniques are designed with the object of providing diversity gains, other than to maximize the post-output SNR at the receiver side, which is usually taken as one of the most important performance figure to minimize the BER performance. Furthermore, more and more wireless communication systems are now equipped with multiple transmit and receive antennas, and therefore it is desired to simultaneously obtain transmit and receive diversity gains so as to combat the severe fading effects.

In this section, we introduce a MRT scheme to fulfill the above two challenges, namely achieving transmit and receive diversity gains and maximizing the post-output SNR (Lo, 1999). Finally, numerical results are presented.

\subsubsection{MRT systems and schemes}

The developed framework of MRT schemes can be regarded as the generalization of MRC for the systems with multiple transmit-and-receive antenna pairs. Figure 9 presents the MRT system model for which $n_{T}$ transmit antennas and $n_{R}$ receive antennas are equipped. Accordingly, there are $n_{T} \times n_{R}$ channel links between the transmitter and the receiver. We assume channel coefficients are statistically independent, as follows:

$$
\mathbf{H}=\left[\begin{array}{ccc}
h_{11} & \cdots & h_{1 n_{T}} \\
\vdots & \ddots & \vdots \\
h_{n_{R} 1} & \cdots & h_{n_{R} n_{T}}
\end{array}\right]=\left[\begin{array}{c}
\mathbf{h}_{1} \\
\vdots \\
\mathbf{h}_{n_{R}}
\end{array}\right]
$$

where the entry $h_{n_{R} n_{T}}$ denotes the flat fading channel gain from the $n_{T} t h$ transmit antenna to the $n_{R}$ th receive antenna. In general, it is essential to acquire the channel state information for successful implementation of a multiple-input multiple-output communication system. The channel estimation issue is beyond the scope of this chapter, and it is assumed that channel state information of (43) is perfectly known at the both transmitter and receiver sides.

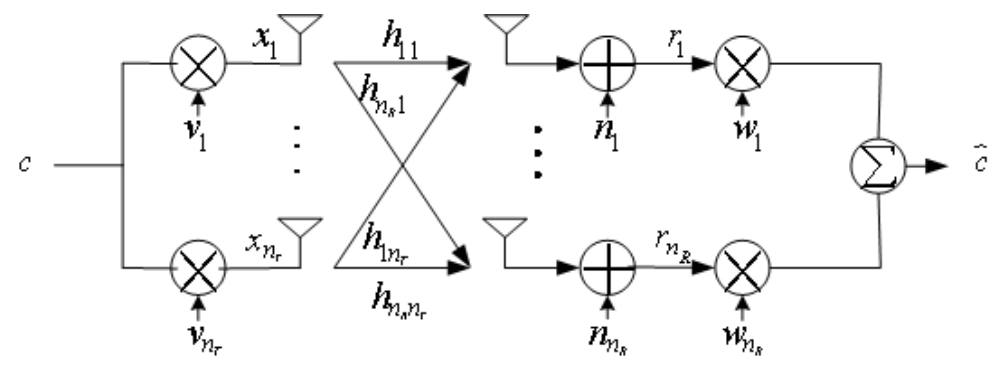

Fig. 9. System model of MRT

The MRT system is a diversity-achieving technique. For each time slot, only one data symbol, denoted by $s$, is transmitted by multiplying with antenna-specific weighting 
coefficients $v_{m}, m=1, \ldots, n_{T}$. The average symbol power is assumed to be $E\left[|s|^{2}\right]=E_{s}$. Denoting the weighting coefficients vector as $\mathbf{v}=\left[v_{1}, \ldots, v_{n_{T}}\right]^{T}$, the signals transmitted by antennas can be expressed by

$$
\mathbf{x}=\left[x_{1}, \ldots, x_{n_{T}}\right]^{T}=s \mathbf{v}
$$

From (43) and (44), the received signals is given by

$$
\mathbf{r}=\mathbf{H} \mathbf{x}+\mathbf{n}
$$

where $\mathbf{n}=\left[n_{1}, \ldots, n_{n_{R}}\right]^{T}$ is the additive white Gaussian noise vector. Subsequently, a weighting vector $\mathbf{w}=\left[w_{1}, \ldots, w_{n_{R}}\right]^{T}$ is applied for combining the received signals at the receive antennas, followed by symbol decision. In the MRT system, we construct the weighting factor $\mathbf{v}$ from the channel matrix $\mathbf{H}$ through a linear transformation as follows:

$$
\mathbf{v}=\frac{1}{a}(\mathbf{g H})^{\dagger}
$$

where $\mathbf{g}=\left[\begin{array}{lll}g_{1} & \cdots & g_{n_{R}}\end{array}\right]$ is a parameter to be determined. The transmitted signal can be alternatively written as

$$
\mathbf{x}=\frac{1}{a}(\mathbf{g H})^{\dagger} \mathrm{s}
$$

where $a$ is a normalization factor such that the transmit power is equal to one, which can be represented as

$$
a=|\mathbf{g H}|=\left(\sum_{p=1}^{n_{R}} \sum_{q=1}^{n_{R}} g_{p} g_{q}^{*} \sum_{k=1}^{n_{T}} h_{p k} h_{g k}^{*}\right)^{1 / 2}
$$

Therefore, from (47), the received signal vector is given by

$$
\mathbf{r}=\frac{1}{a} \mathbf{H}(\mathbf{g H})^{\dagger} s+\mathbf{n}
$$

In order to make symbol decision, the weighting vector $\mathbf{w}$ is applied to combine the received signal vector $\mathbf{x}$ for signal reception. If $\mathbf{w}^{T}$ is equal to $\mathbf{g}$, the output is given by

$$
\tilde{s}=\mathbf{w}^{T} \mathbf{r}=\mathbf{g} \frac{1}{a} \mathbf{H}(\mathbf{g H})^{\dagger} s+\mathbf{g n}=a s+\mathbf{g n}
$$

The post-output SNR after combining is thus calculated as

$$
\gamma=\frac{a^{2}}{\mathbf{g g}^{\dagger}} \gamma_{0}=\frac{a^{2} \gamma_{0}}{\sum_{p=1}^{n_{R}}\left|g_{p}\right|^{2}}
$$


where $\gamma_{0}=E_{s} / \sigma_{n}^{2}$ denotes the average SNR for a single transmit antenna. It is observed from (51) that the post-output SNR only depends on the value of $\mathbf{g}$. Thus, to maximize the post-output SNR is equivalent to choosing an appropriate value of $\mathbf{g}$. By assuming all channel links between the transmitter and receiver are statistically identical, each of the transmitter antenna has to be allocated with the same weighting power; that is, we have $\left|g_{1}\right|=\left|g_{2}\right|=\cdots=\left|g_{n_{R}}\right|$. Without loss of generality, we can set $\left|g_{i}\right|=1$, for $i=1, \cdots, n_{R}$, for simplicity. Accordingly, the post-output SNR in (51) is simplified as

$$
\gamma=\frac{a^{2}}{N} \gamma_{0}
$$

By applying the Cauchy-Schwarz inequality, we can further get that $a^{2}$ is maximized, i.e., maximizing the post-output SNR, if and only if we set

$$
\left(g_{p} g_{q}^{*}\right)^{*}=\frac{\sum_{k=1}^{n_{T}} h_{p k} h_{g k}^{*}}{\left|\sum_{k=1}^{n_{T}} h_{p k} h_{g k}^{*}\right|}
$$

It is noted that the denominator term in (53) is due to the fact of $\left|g_{i}\right|=1$ and $\left|g_{p} g_{q}^{*}\right|=1$. In accordance with (52) and (53), the maximum post-output SNR is given by

$$
\begin{aligned}
\gamma_{\max } & =\frac{\gamma_{0}}{N} \sum_{p=1}^{n_{R}} \sum_{q=1}^{n_{R}}\left|\sum_{k=1}^{n_{T}} h_{p k} h_{g k}^{*}\right| \\
& =\frac{\gamma_{0}}{N} \sum_{p=1}^{n_{R}} \sum_{q=1}^{n_{R}}\left|\mathbf{h}_{p} \mathbf{h}_{q}^{\dagger}\right|
\end{aligned}
$$

On the one hand, if $\mathbf{h}_{p}$ and $\mathbf{h}_{q}$ are mutually orthogonal, i.e., $\mathbf{h}_{p} \mathbf{h}_{q}^{H}=0, \gamma_{\max }$ takes the minimum value, given by

$$
\gamma_{\max }=\frac{\gamma_{0}}{N} \sum_{p=1}^{n_{R}} \sum_{k=1}^{n_{T}}\left|h_{p k}\right|^{2}
$$

On the other hand, if $\mathbf{h}_{p}$ and $\mathbf{h}_{q}$ are fully correlated, i.e., $\mathbf{h}_{p} \mathbf{h}_{q}^{H}=\left|\mathbf{h}_{q}\right|^{2}, \gamma_{\max }$ takes the maximum value, given by

$$
\gamma_{\max }=\frac{\gamma_{0}}{N} \sum_{p=1}^{n_{R}} \sum_{q=1}^{n_{R}} \sum_{k=1}^{n_{T}}\left|h_{p k}\right|^{2}
$$

Form (55) and (56), the average post-output SNR is therefore bounded by

$$
M \bar{\gamma}_{0} \leq \bar{\gamma}_{\max } \leq N M \bar{\gamma}_{0}
$$

where $\bar{\gamma}_{0}=\gamma_{0} E\left[\left|h_{p k}\right|^{2}\right]$ is defined as the average SNR at each branch. Also, for an MRT system with $n_{T} \times n_{R}$ antennas, it is expected from (55) that the diversity order is equal to $n_{T} \times n_{R}$. 


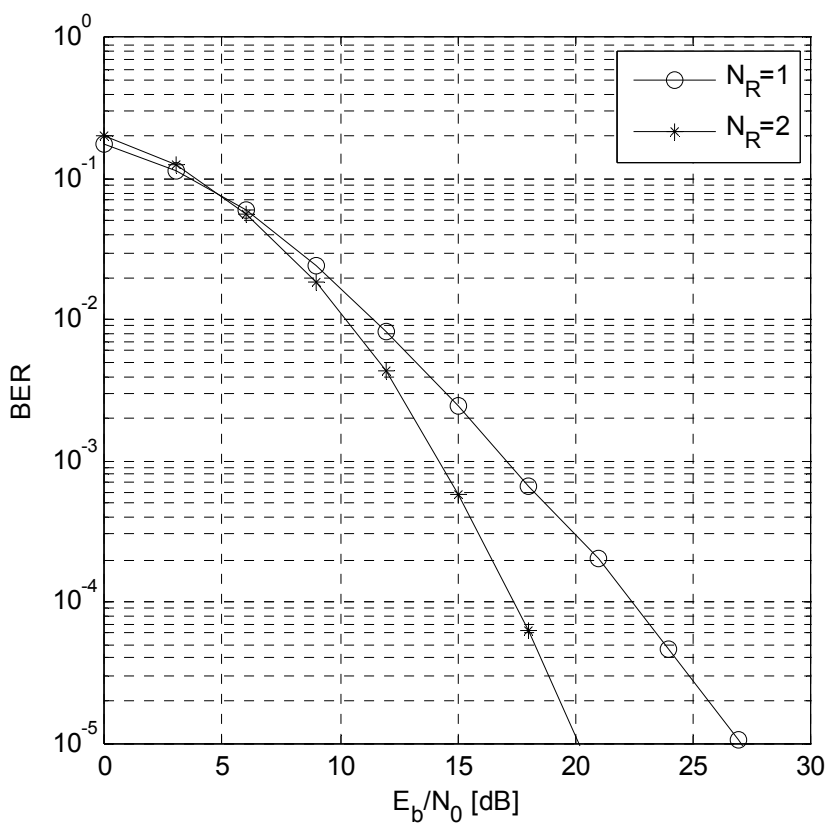

Fig. 10. Comparison of MRT schemes with $\mathrm{N}_{T}=2$ and QPSK modulation

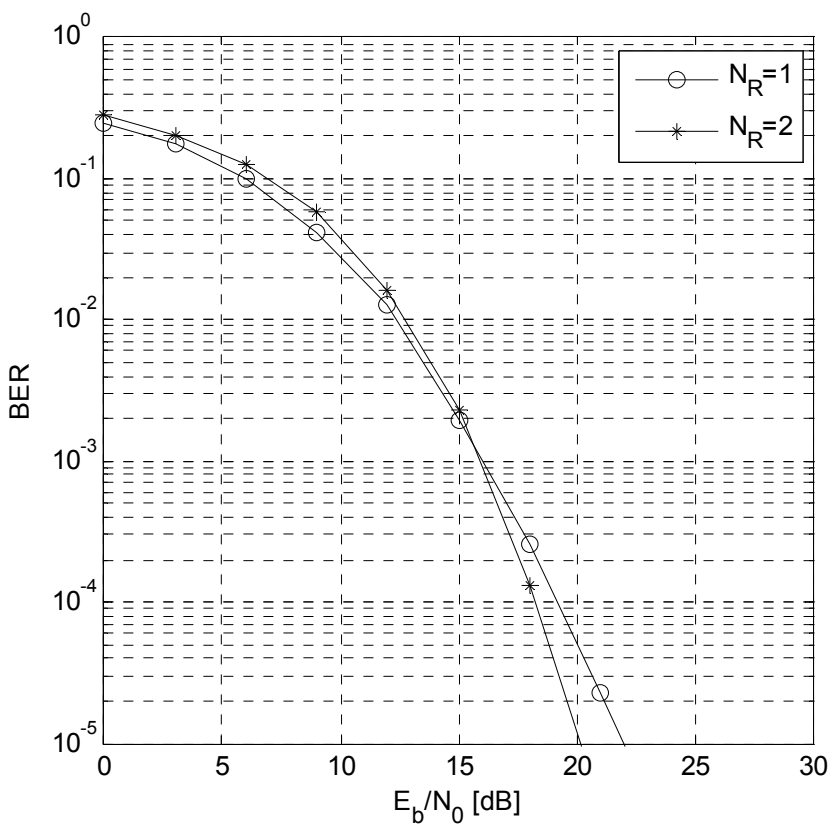

Fig. 11. Comparison of MRT schemes with $\mathrm{N}_{T}=4$ and QPSK modulation 


\subsubsection{BER performance of MRT scheme}

The BER performance of the MRT scheme is simulated as follows. At the beginning, we assume that the complex Gaussian fading gains among transmit-receive pairs are mutually independent. The total channel power from all the transmit antennas to a receive antenna is normalized to one. The QPSK modulation is adopted in the simulation. Moreover, it is assumed that channel fading gains are perfectly available to both transmitter and receiver sides. Fig. 10 and Fig. 11 demonstrate the BER performance curves for an MRT system with two transmit antennas and two and four receive antennas, respectively. It is observed from these two figures that the BER performance can be improved as the number of transmit and receive antennas increases, and we can gain much diversity gains by increasing the number of antennas.

\section{Spatial multiplexing techniques}

Recent research resutls from information theory aspects have disclosed that rich scattering wireless channels can provide enormous capacity which increases proportional to the minimum number of transmit and receive antennas (Foschini, 1996). Spatial multiplexing based MIMO (SM-MIMO) systems, in which multiple data streams are simultaneously transmitted through multiple antennas, is a new attractive technique to realize high data rate transmission, while signal detection at the receiver is a difficult task for SM-MIMO systems. Although the well-known ML detector can be applied for the SM-MIMO systems achieve the best performance, its complexity increases exponentially as the number of data stream and the number of transmit antennas increase.

Foschini proposed a layered space-time (LST) or Bell laboratories layed space-time (BLAST) architecture (Foschini \& Gans, 1998), allowing for processing multi-dimensional received signals in spatial domain. At the transmitter, multiple data streams are first encoded and then distributed over multiple antennas. At the reciever, the received signals are separated and subsequently decoded by integrating interference supression or cancellation tehcniques with decoding algorithms, thus leading to much lower computation complexity, as compared with the ML decoding. Various BLAST architectures, depending on whether error control coding is adopted or not, have been investigated, e.g., horizontal BLAST (H-BLAST), diagonal BLAST (D-BLAST), and vertical BLAST (V-BLAST). The error control coding is applied for both the H-BLAST and D-BLAST architectures, while the V-BLAST does not consider the use of error control coding. Although the H-BLAST and D-BLAST architectures can gain better performance due to the coding gains in spatial domain, they suffer from a spectral efficiency loss and higher implementation complexity (Foschini \& Gans, 1998). Therefore, the uncoded and simplified V-BLAST architecture, proposed in (Wolniansky et al., 1998), is considered as an effective solution to fullfill the potential spatial multiplexing gains. In this section, we focus on the V-BLAST architecture, followed by data detection techniques used to separate and detect the V-BLAST signals. Two essential V-BLAST receivers based on ZF and MMSE are introduced. Finally, we discuss the BER performance of the V-BLAST architecture with different data detection algorithms.

\subsection{V-BLAST systems}

The block diagram of the V-BLAST system is presented in Fig. 12, where there are $n_{T}$ transmit and $n_{R}$ receive antennas and $n_{T} \leq n_{R}$. A single data stream is divided into $n_{T}$ substreams, each of which is then individually modulated into symbols and transmitted 
from its corresponding transmit antenna. Without loss of generality, it is assumed that the same constellation is applied for all substreams, and the transmission duration is a burst consisting of $L$ symbols. We assume that the multiple-input multiple-output channel is flat fading and quasi-static over the duration of $L$ symbols, and the channel matrix is denoted by $\mathbf{H}_{n_{R} \times n_{T}}$, whose $(i, j)$ th entry $h_{i j}$ represents the complex channel gain from the $j t h$ transmit antenna to the $i t h$ receive antenna. We denote the data vector to be transmitted as

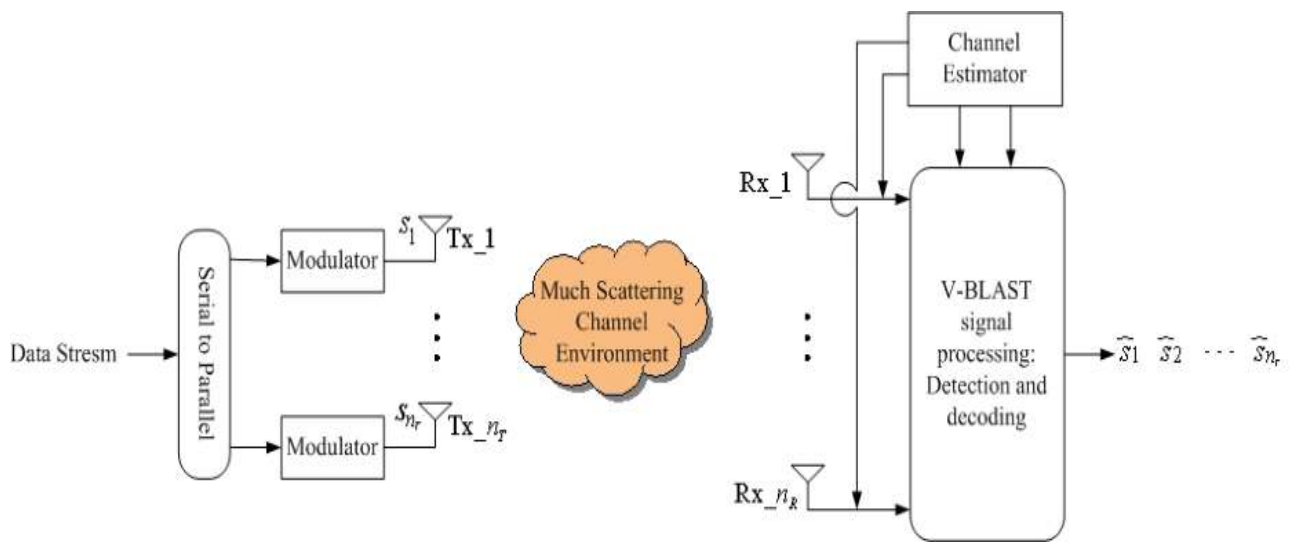

Fig. 12. Block diagram of V-BLAST systems

$$
\mathbf{s}=\left[s_{1}, \ldots, s_{n_{T}}\right]^{T}
$$

where $s_{i}$ is the modulated symbol at the $i$ th transmit antenna. The received signals at the $n_{R}$ receive antennas can thus be represented as a vector, as follows:

$$
\mathbf{r}=\mathbf{H s}+\mathbf{n}
$$

where $\mathbf{n}$ is an independent and identical distributied (i.i.d.) white Gaussian noise vector with zero mean and a covariance matrix $\sigma_{n}^{2} \mathbf{I}$.

\subsection{V-BLAST data detection}

The idea to perform data detection for this system is to incorporate conventional linear equalizers with nonlinear interference cancellation methods. Each substream is in trun detected, and the reminders are considered as interference signals. The equalizer is designed according to some specific criteria, e.g., ZF and MMSE, to null out the interference signals by linearly weighting the received signals with equalizer coefficients. In the following, we discuss the ZF-based V-BLAST receiver and the MMSE-based V-BLAST receiver.

\subsubsection{ZF-based V-BLAST algorithm}

First of all, the ZF nulling is performed by strictly choosing nulling vectors $\mathbf{w}_{i}$, for $i=1, \ldots, M$, such that

$$
\mathbf{w}_{i}^{\dagger}(\mathbf{H})_{j}=\delta_{i j}, \text { for } i, j=1,2, \cdots n_{T}
$$


where $(\mathbf{H})_{j}$ denotes the $j$ th column of $\mathbf{H}$, the notation $(\cdot)^{\dagger}$ takes the Hermitian, and $\delta$ is the Kronecker delta function. Then, the $i$ th substream after equalization is given by

$$
y_{i}=\mathbf{w}_{i}^{\dagger} \mathbf{r}
$$

The ZF-based detection procedures to extract substreams for any arbitrary detection order are elaborated in the following. Let us set $j=1, \mathbf{r}_{1}=\mathbf{r}$ and the ordering set for data detection as $\varsigma=\left\{k_{1}, k_{2}, \cdots, k_{n_{T}}\right\}$, where $k_{j} \in\left\{1, \ldots, n_{T}\right\}$.

Step 1. Use the nulling vector $\mathbf{w}_{k_{j}}$ to obtain the decision statistic for the $k_{j}$ th substream

$$
y_{k_{j}}=\mathbf{w}_{k_{j}}^{T} \mathbf{r}_{j}
$$

Step 2. Slice $y_{k_{j}}$ to obtain the hard decision $\hat{s}_{k_{j}}$

$$
\hat{s}_{k_{j}}=Q\left(y_{k_{j}}\right)
$$

where $Q(\bullet)$ denotes the hard decision operation.

Step 3. Reconstruct and cancel out the currently detected substream from the received signal $\mathbf{r}_{j}$, resulting in a modified received signal vector $\mathbf{r}_{j+1}$

$$
\mathbf{r}_{j+1}=\mathbf{r}_{j}-\hat{s}_{k_{j}}(\mathbf{H})_{k_{j}}
$$

Update $\mathrm{j}$ to $\mathrm{j}+1$ for the next iteration, and repeat the Step1 Step3, where the $k_{j}$ th ZF nulling vector is given by

$$
\mathbf{w}_{k_{j}}^{\dagger}(\mathbf{H})_{k_{i}}= \begin{cases}0, & \text { for } i \geq j \\ 1, & \text { for } i=j\end{cases}
$$

Thus, if the inter-antenna interference is perfectly reconstructed and cancelled, the weighting vector $\mathbf{w}_{k_{j}}^{\dagger}$ is orthogonal to the subspace spanned by the interference-reduced vector $\mathbf{r}_{j+1}$. Accordingly, the solution to $\mathbf{w}_{k_{j}}^{\dagger}$ in (65) is the $k_{j}$ th row of $\widetilde{\mathbf{H}}_{j-1}^{+}$, where the notation $\widetilde{\mathbf{H}}_{j-1}$ denotes the matrix acquired by deleting columns $k_{1}, k_{2}, \cdots, k_{j-1}$ of $\mathbf{H}$ and $(\cdot)^{+}$ denotes the Moore-Penrose pseudoinverse (Abadir \& Magnus, 2006). The post-detection SNR for the $k_{j}$ th substream of $\mathbf{s}$ is therefore given by

$$
S N R_{k_{j}}=\frac{E_{S}}{\sigma_{n}^{2}\left\|\mathbf{w}_{k_{j}}\right\|^{2}}
$$

where $E_{s}=E\left[\left|s_{k_{j}}\right|^{2}\right]$

\subsubsection{MMSE-based V-BLAST algorithm}

The MMSE is another well-known criterion for designing V-BLAST data detection. For the MMSE criterion, we intend to find the equalizer coefficients to minimize the mean squared error between the transmitted vector $\mathbf{s}$ and the equalized output $\mathbf{W}^{\dagger} \mathbf{r}$, as follows: 


$$
\mathbf{W}_{\text {MMSE }}=\arg \min _{\mathbf{W}} E\left\{\left(\mathbf{s}-\mathbf{W}^{\dagger} \mathbf{r}\right)^{2}\right\}
$$

The optimal MMSE equalizer $\mathbf{W}_{M M S E}$ is expressed as

$$
\mathbf{W}_{M M S E}=\left(\mathbf{H H}^{\dagger}+\sigma_{n}^{2} \mathbf{I}_{n_{R}}\right)^{-1} \mathbf{H}
$$

where $\sigma_{n}^{2}$ denotes the noise power and $\mathbf{I}_{n_{R}}$ represents an $n_{R} \times n_{R}$ identity matrix. Thus, the decision statistic for the $i$ th substream is given by

$$
y_{i}=\left(\mathbf{W}_{M M S E}\right)_{i}^{\dagger} \mathbf{r}
$$

where $\left(\mathbf{W}_{M M S E}\right)_{i}$ denotes the ith column of the matrix $\mathbf{W}_{M M S E}$. Subsequently, the hard decision for the ith substream is given by

$$
\hat{s}_{i}=Q\left(y_{i}\right)
$$

To further improve the performance, one can incorporate the interference cancellation methodology, similar to the idea in subsection 3.2.1, into the MMSE equalizer. Concerning an arbitrary detection order, the interference suppression and cancellation procedure is the same as the Step1 Step3 in subsection 3.2.1, but the MMSE equalizer is used instead of the nulling vector as follows. Define the MMSE equalizer at the $j$ th iteration as $\mathbf{W}_{M M S E}^{j}$ :

$$
\mathbf{W}_{M M S E}^{j}=\left(\mathbf{H}_{d}^{j-1}\left(\mathbf{H}_{d}^{j-1}\right)^{\dagger}+\sigma_{n}^{2} \mathbf{I}_{n_{R}}\right)^{-1} \mathbf{H}_{d}^{j-1}
$$

where $\mathbf{H}_{d}^{j-1}$ represents the truncated channel matrix by deleting the columns $k_{1}, k_{2}, \cdots, k_{j-1}$ of $\mathbf{H}$. At the $j$ th iteration, the weighting vector for detecting the $k_{j} t h$ substream, $\left(\mathbf{W}_{M M S E}^{j}\right)_{k_{j}}$, is thus obtained from the $k_{j}$ th column of $\mathbf{W}_{M M S E}^{j}$.

\subsubsection{Ordering V-BLAST algorithm}

Since the ZF-based and MMSE-based V-BLAST data detection algorithms iterate between equalization and interference cancellation, the order for detecting the substreams of $\mathbf{s}$ becomes an important role to determine the overall performance (Foschini et al., 1999). In this subsection, we discuss an ordering scheme for the two V-BLAST detectoin algorithms. Although the ZF or MMSE equalizer can null out or supress the residual inter-antenna interference, it will introudce the noise enhancement problem, leading to incorrect data decision, and the incorrect interference reconstruction will cause the error propagation problem. Assuming that all the substreams adopt the same constellation scheme, among all the remaining entries of $\mathbf{s}$ (not yet detected), the entry with the largest SNR, i.e., from (66), having the minimum norm power $\left\|\mathbf{w}_{k_{j}}\right\|^{2}$, is choosen at each iteration in the detection process. The iterative procedures for the ordering ZF-based or MMSE-based V-BLAST detection algorithms are described in the following. 


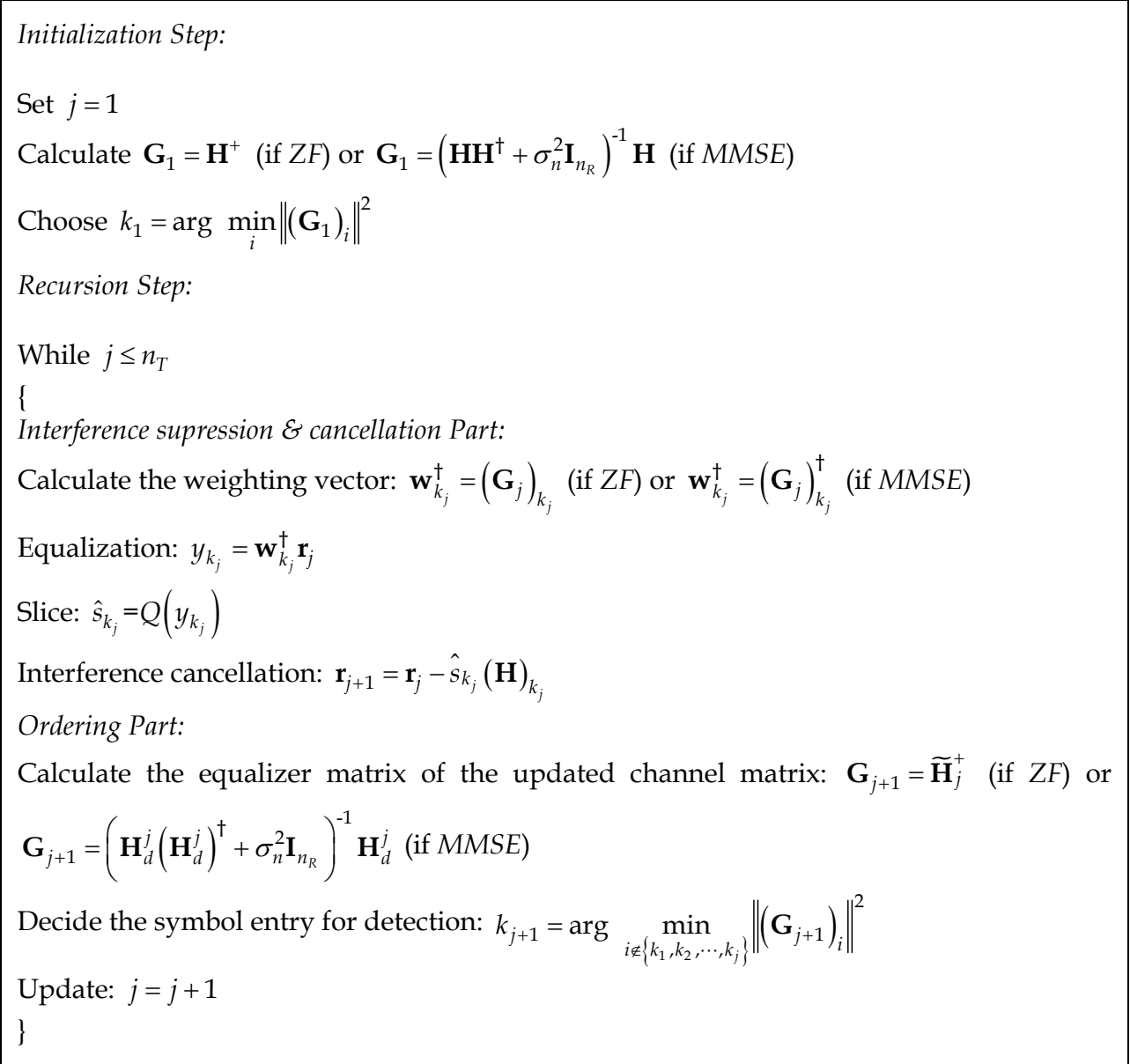

In the above iterative procedure, for the $\mathrm{ZF}$ case, the vector $\left(\mathbf{G}_{j}\right)_{i}$ denotes the $i t h$ row of the matrix $\mathbf{G}_{j}$, computed from the pseudoinverse of $\widetilde{\mathbf{H}}_{j-1}$, where the columns $k_{1}, \cdots, k_{j-1}$ are set to zero. However, for the MMSE case, the vector $\left(\mathbf{G}_{j}\right)_{i}$ denotes the the ith column of the matrix $\mathbf{G}_{j}$, computed from the MMSE equalizer of $\mathbf{H}_{d}^{j-1}$, where the columns $k_{1}, \cdots, k_{j-1}$ are set to zero. This is because these columns only related to the entries of $s_{k_{1}}, \cdots, s_{k_{j}}$ which have already been estimated and cancelled. Thus, the system can be regarded as a degenerated V-BLAST system of Figure 12 where the transmitters $k_{1}, \cdots, k_{j}$ are removed.

\subsubsection{BER Performance of various V-BLAST detection algorithms}

The BER performance of the V-BLAST with ML, ZF, and MMSE algorithms is presented in the following. Both the transmitter and the receiver are equipped with four antennas, and a flat fading channel is used for simulation. Fig. 13 compares the BER performance of the ML detector with those of the ZF-based or MMSE-based V-BLAST algorithms without ordering, in which the detecting order is in sequence from the last transmit antenna to the first 
transmit antenna. As compared with the ZF-based and MMSE-based V-BLAST algorithms, the ML detector has better BER performance. However, the computation complexity of the ML detector exponentially increases as the modulation order or the number of transmit antennas increase. Instead, the ZF-based and MMSE-based V-BLAST algorithms, which are the linear detection methods combined with the interference cancellation methods, require much lower complexity than the ML detector, but their BER performance is significantly inferior to that of the ML detector.

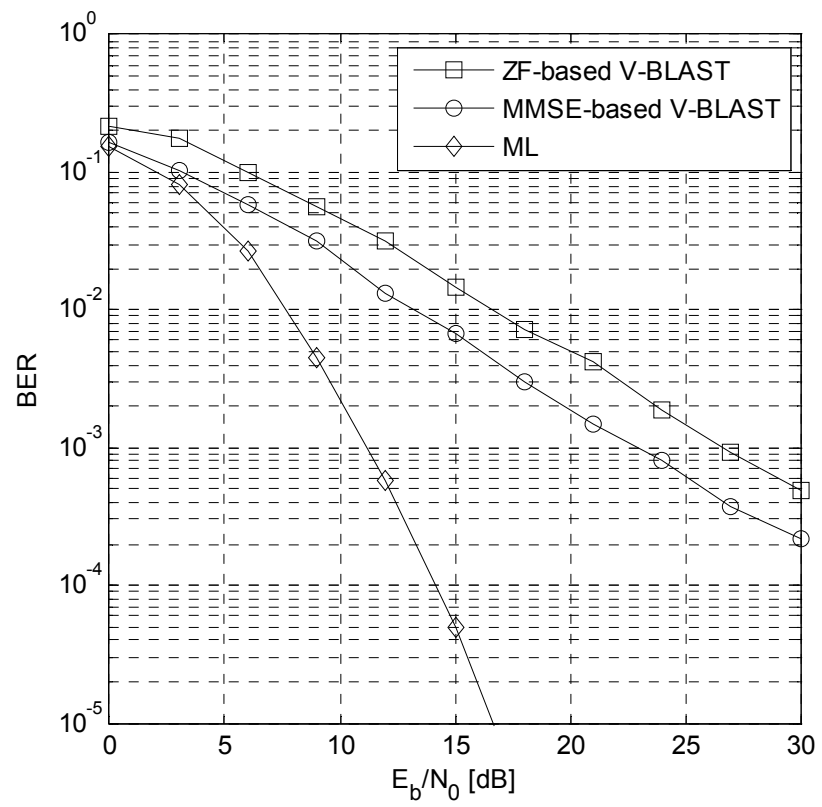

Fig. 13. BER performance of $4 \times 4$ V-BLAST systems without ordering

Fig. 14 and Fig. 15 demonstrate the BER performance of the ZF-based and the MMSE-based V-BLAST algorithm, respectively, with or without ordering. We can observe from these two figures that the V-BLAST algorithms with ordering can achieve better performance than that of the algorithms without ordering. An ordered successive interference suppression and cancellation method can effectively combat the error propagation problem to improve the BER performance with less complexity, although the ML detector still outperforms the ordering V-BLAST algorithms.

\section{Beamforming}

Beamforming is a promising signal processing technique used to control the directions for transmitting or receiving signals in spatial-angular domain (Godara, 1997). By adjusting beamforming weights, it can effectively concentrate its transmission or reception of desired signals at a particular spatial angle or suppress unwanted interference signals from other spatial angles. In this section, we will introduce the general concepts of beamforming techniques as well as some famous beamforming methods. 


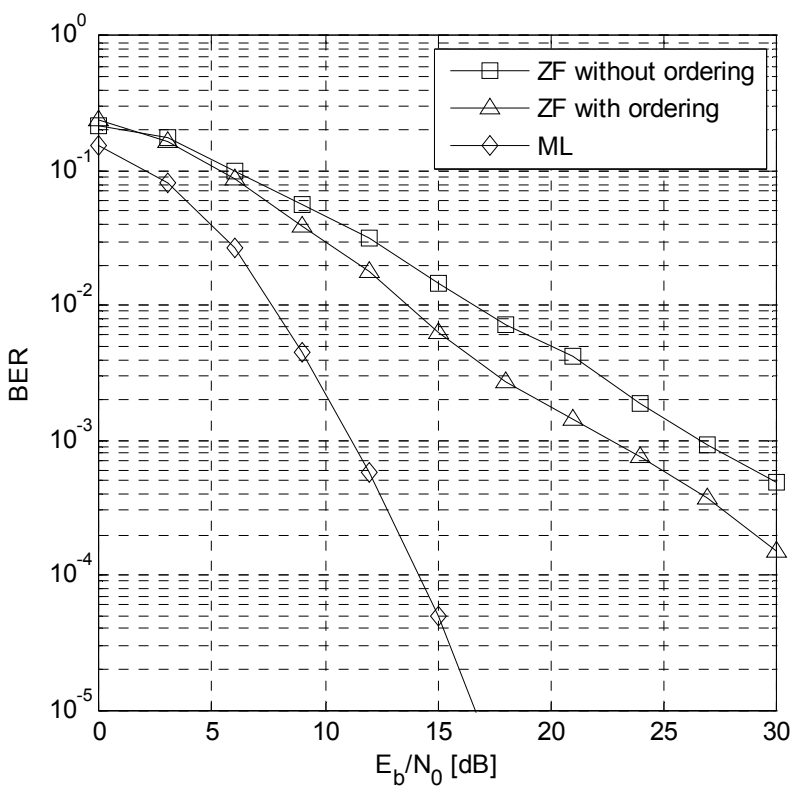

Fig. 14. BER performance of $4 \times 4$ V-BLAST systems using ZF-based V-BLAST algorithm with or without ordering

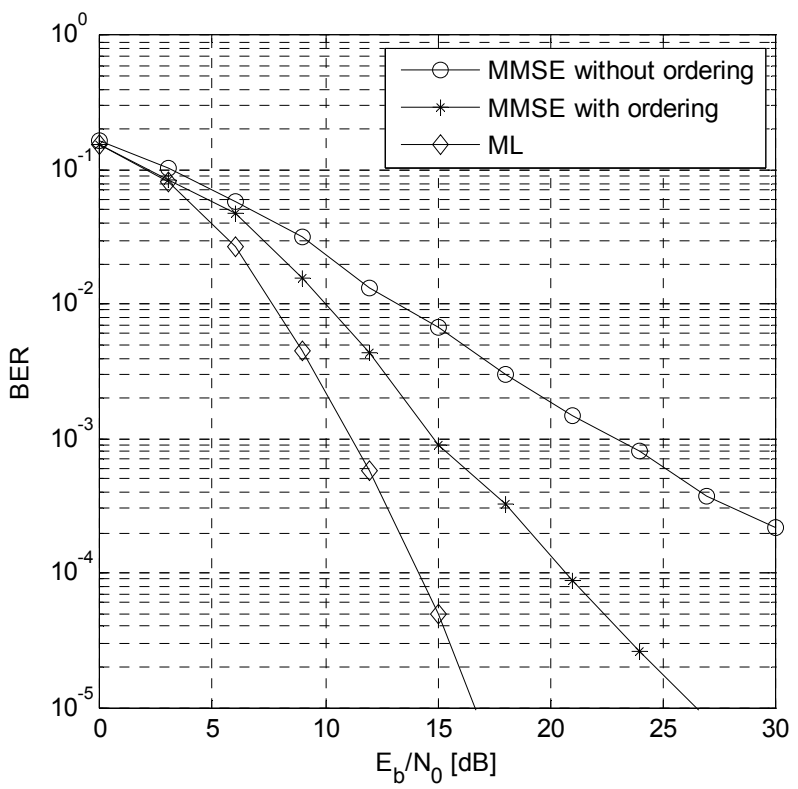

Fig. 15. BER performance of $4 \times 4 \mathrm{~V}$-BLAST systems using MMSE-based V-BLAST algorithm with or without ordering 


\subsection{Linear array and signal model}

Fig. 16 depicts a linear array with $L$ omni-directional and equi-spaced antennas, and the antenna spacing is set as $d$.

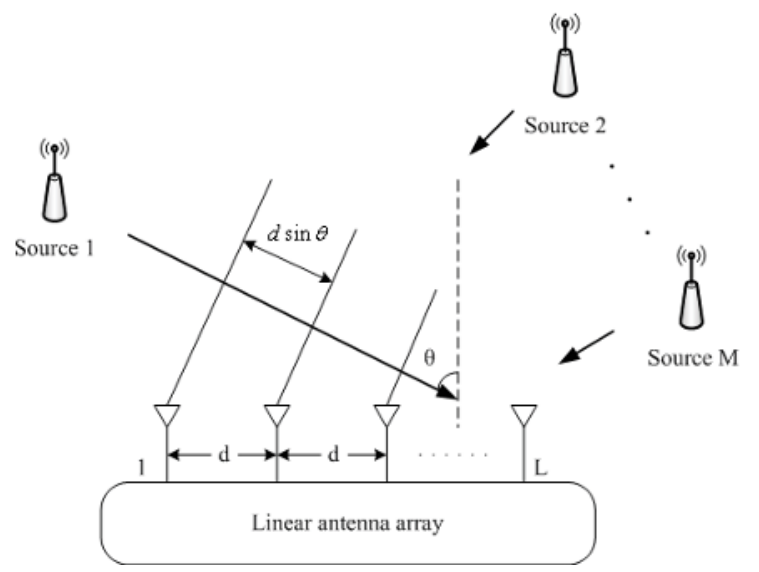

Fig. 16. Linear array

Let the first antenna element of the linear array be the reference point at the origin of the $x-y$ coordinate system. Consider $M$ uncorrelated far-field sources with the same central carrier frequency $f_{0}$, for $i=1, \ldots, M$, and due to the far-field assumption, each source can be approximated as a plane wave when arriving at the linear array. Accordingly, the time arrival of the plane wave from the $i t h$ source in the direction of $\theta_{i}$ to the $l$ th antenna is given by

$$
\tau_{l}\left(\theta_{i}\right)=\frac{d}{c}(l-1) \sin \theta_{i}
$$

where $c$ is the speed of light. Denote $m_{i}(t)$ as the baseband signal of the $i t h$ source. By assuming that the bandwidth of the source is narrow enough, i.e., $m_{i}\left(t+\tau_{l}\left(\theta_{i}\right)\right) \cong m_{i}(t)$, the bandpass signal on the $l$ th antenna contributed by the $i t h$ source is expressed as

$$
\begin{aligned}
b_{i}(t) & =\mathfrak{R e}\left\{m_{i}\left(t+\tau_{l}\left(\theta_{i}\right)\right) e^{j 2 \pi f_{0}\left(t+\tau_{l}\left(\theta_{i}\right)\right)}\right\} \\
& \cong \mathfrak{R e}\left\{m_{i}(t) e^{j 2 \pi f_{0}\left(t+\tau_{l}\left(\theta_{i}\right)\right)}\right\}
\end{aligned}
$$

Thus, the corresponding baseband equivalent signal on the $l$ th antenna is given by

$$
m_{i}(t) e^{j 2 \pi f_{0} \tau_{l}\left(\theta_{i}\right)}
$$

Let $x_{l}(t)$ denote the baseband representation of the received signal on the lth antenna, including both $M$ sources and noise on the $l$ th antenna, and from (74), it is given by

$$
x_{l}(t)=\sum_{i=1}^{M} m_{i}(t) e^{j 2 \pi f_{0} \tau_{l}\left(\theta_{i}\right)}+n_{l}(t)
$$


where $n_{l}(t)$ is the spatially additive white Gaussian noise term on the lth antenna with zero mean and variance $\sigma_{n}^{2}$.

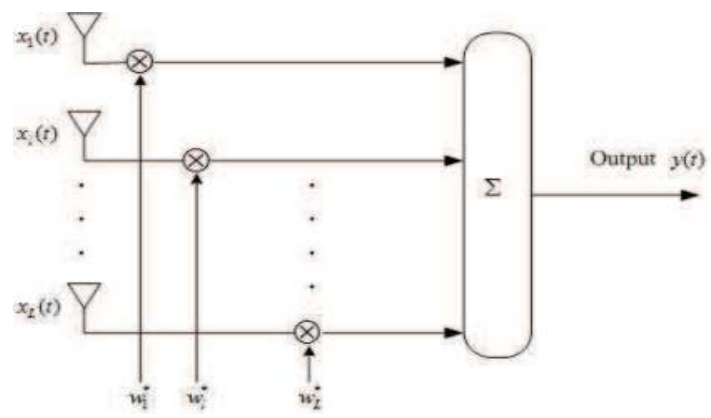

Fig. 17. Antenna beamforming

Fig. 17 shows the spatial signal processing of a beamforming array, in which complex beamforming weights $w_{l}$ are applied to produce an output of linear combination of the received signals $x_{l}(t)$, expressed as

$$
y(t)=\sum_{l=1}^{L} w_{l}^{*} x_{l}(t)
$$

Define $\mathbf{w}=\left[w_{1}, w_{2}, \ldots, w_{L}\right]^{T}$ and $\mathbf{x}(t)=\left[x_{1}(t), x_{2}(t), \ldots, x_{L}(t)\right]^{T}$. From (75), we can express $\mathbf{x}(t)$ in a matrix-vector form as follows

$$
\mathbf{x}(t)=\sum_{i=1}^{M} m_{i}(t) \mathbf{a}_{i}\left(\theta_{i}\right)+\mathbf{n}(t)
$$

where $\mathbf{n}(t)=\left[n_{1}(t), n_{2}(t), \ldots, n_{L}(t)\right]^{T}$, and $\mathbf{a}_{i}\left(\theta_{i}\right)=\left[e^{j 2 \pi f_{0} \tau_{1}\left(\theta_{i}\right)}, \ldots, e^{j 2 \pi f_{0} \tau_{L}\left(\theta_{i}\right)}\right]^{T}$ is known as the steering vector for the ith source. Then, we can rewrite (77) into a matrix notation, leading to a compact representation:

$$
y(t)=\mathbf{w}^{\dagger} \mathbf{x}(t)
$$

From (77) and (78), for a given beamforming vector, the mean output power is calculated as

$$
P(\mathbf{w})=E\left[y(t) y^{*}(t)\right]=\mathbf{w}^{\dagger} \mathbf{R} \mathbf{w}
$$

where $E[\cdot]$ denotes the expectation operator and $\mathbf{R}$ is the correlation matrix of the signal $\mathbf{x}(t)$, which is defined and given by

$$
\mathbf{R}=E\left[\mathbf{x}(t) \mathbf{x}^{\dagger}(t)\right]=\sum_{i=1}^{M} p_{i} \mathbf{a}_{i} \mathbf{a}_{i}^{\dagger}+\sigma_{n}^{2} \mathbf{I}
$$

where $p_{i}$ is the power of the $i$ th source, $\mathbf{I}$ is an identity matrix of size $L \times L$. In the following, we introduce three famous beamforming schemes to determine the complex beamforming weights. 


\subsection{Conventional beamforming}

A conventional beamforming scheme is to form a directional beam by merely considering a single source. Since all its beamforming weights are set with an equal magnitude, this scheme is also named as delay-and-sum beamforming. Without loss of generality, assume that the targeted source for reception is in the direction of $\theta_{0}$, and the beamforming weight vector is simply given by

$$
\mathbf{w}=\frac{1}{L} \mathbf{a}\left(\theta_{0}\right)
$$

Now we consider a communication environment consisting of only one signal source, and with this delay-and-sum beamforming scheme, the output signal after beamforming is given by

$$
y(t)=\mathbf{w}^{\dagger} \mathbf{x}(t)=\mathbf{w}^{\dagger} \mathbf{a}\left(\theta_{0}\right) m_{0}(t)+\mathbf{w}^{\dagger} \mathbf{n}(t)
$$

By assuming that the power of the source signal is equal to $p_{0}$, the post-output SNR after beamforming is then calculated as

$$
S N R=\frac{\left\|\mathbf{w}^{\dagger} \mathbf{a}\left(\theta_{0}\right) m_{0}(t)\right\|^{2}}{E\left[\left\|\mathbf{w}^{\dagger} \mathbf{n}(t)\right\|^{2}\right]}=\frac{L p_{0}}{\sigma_{n}^{2}}
$$

In fact, the conventional beamforming can be regarded as an MRC-like scheme, as introduced in (Godara, 1997). It can be easily proved that for the case of a single source, the conventional beamforming scheme can provide the maximum output SNR. We can also observe that the output SNR in (83) is proportional to the number of antenna arrays, and as the number of antennas increases, it can facilitate to reduce the noise effect. However, when there are a number of signal sources which can interfere with each other, this conventional beamforming scheme does not have ability to suppressing interference effectively.

\subsection{Null-steering beamforming}

Consider a communication environment consisting of one desired signal source with an angle of arrival $\theta_{0}$ and multiple interfering signal sources with angles of arrival $\theta_{i}$, for $i=1, \ldots, M$. To effectively mitigate the mutual interference, a null-steering beamforming scheme can be designed to null out unwanted signals from some interfering sources with known directions. The null-steering beamforming scheme is also named as $\mathrm{ZF}$ beamforming. As its name suggested, the design idea is to form beamforming weights with unity response in the desired source direction $\theta_{0}$, while create multiple nulls in the interfering source directions $\theta_{i}$, for $i=1, \ldots, M$. Now assume that the steering vector for the desired and interfering signal sources are respectively denoted by $\mathbf{a}_{0}$ and $\mathbf{a}_{i}$, for $i=1, \ldots, M$. Then, the beamforming weights can be designed by solving the following equations ( $\mathrm{D}^{\prime}$ Assumpcao \& Mountford, 1984):

$$
\begin{aligned}
& \mathbf{w}^{\dagger} \mathbf{a}_{0}=1 \\
& \mathbf{w}^{\dagger} \mathbf{a}_{i}=0, \text { for } i=1, \cdots, M
\end{aligned}
$$


Using the matrix notation, we can rewrite (84) as follows

$$
\mathbf{w}^{\dagger} \mathbf{A}=\mathbf{e}_{1}^{T}
$$

where $\mathbf{A}$ is a matrix containing $M+1$ steering vectors, i.e., $\mathbf{A}=\left[\mathbf{a}_{0}, \mathbf{a}_{1}, \ldots, \mathbf{a}_{M}\right]$, and we define $\mathbf{e}_{1}=[1,0, \cdots, 0]^{T}$. If the total number of desired and interfering sources is equal to the number of antennas, $\mathbf{A}$ is an invertible square matrix as long as $\theta_{i} \neq \theta_{j}$, for all $i, j$. As a result, the solution for the beamforming weights is given by

$$
\mathbf{w}^{\dagger}=\mathbf{e}_{1}^{T} \mathbf{A}^{-1}
$$

Otherwise, the solution is obtained via taking the pseudo inverse of $\mathbf{A}$. The solution for the beamforming weights becomes

$$
\mathbf{w}^{\dagger}=\mathbf{e}_{1}^{T} \mathbf{A}^{\dagger}\left(\mathbf{A} \mathbf{A}^{\dagger}\right)^{-1}
$$

It is noted that although this beamforming scheme has nulls in the directions of interfering sources, it is not designed to maximum the output SNR. Hence, it is not an optimal one from the viewpoint of maximizing the output SNR.

\subsection{Optimal beamforming}

Although the null-steering beamforming scheme can deal with the interference problem by nulling out unwanted signals, it suffers from two critical problems. One is that the nullbeamforming scheme requires the knowledge of interfering source directions, which is not easily acquired in practice. The other is that the output SNR is not the maximum, even though this scheme is quite simple. To overcome these two problems in the environment comprising of one desired signal source with the steering vector $\mathbf{a}_{0}$ and $M$ interfering sources with the steering vectors $\mathbf{a}_{i}$, for $i=1, \ldots, M$, from (79), we can derive the optimal beamforming weights by minimizing the total output power, under the constraint that the output gain in the direction of desired signal source is equal to one; that is

$$
\begin{aligned}
& \min _{\mathbf{w}} \mathbf{w}^{\dagger} \mathbf{R w} \\
& \text { subject to } \mathbf{w}^{\dagger} \mathbf{a}_{0}=1
\end{aligned}
$$

where $\mathbf{R}=\sum_{i=0}^{M} p_{i} \mathbf{a}_{i} \mathbf{a}_{i}^{\dagger}+\mathbf{R}_{n}, p_{i}$ is the signal power of the corresponding source, and $\mathbf{R}_{n}$ is the covariance matrix of the spatial noise. Here, we consider a more general case where the noise is not necessary spatial white. This optimal beamforming scheme is also known as minimum variance distortionless response (MVDR) beamforming. The Lagrange multiplier can be applied to solve this optimization problem, and the Lagrange function associated with (88) is given by

$$
L(\mathbf{w}, v)=\mathbf{w}^{\dagger} \mathbf{R} \mathbf{w}+v\left(\mathbf{w}^{\dagger} \mathbf{a}_{0}-1\right)=\mathbf{w}^{\dagger}\left(\mathbf{R} \mathbf{w}+v \mathbf{a}_{0}\right)-v
$$

where $v$ is the Lagrange multiplier. By applying the Karush-Kuhn-Tucker (K.K.T.) conditions, the sufficient conditions to reach the optimal solution are given by 


$$
\begin{gathered}
\nabla_{\mathbf{w}} L(\mathbf{w}, v)=2 \mathbf{R} \mathbf{w}+v \mathbf{a}_{0}=0 \\
\mathbf{w}^{\dagger} \mathbf{a}_{0}=1
\end{gathered}
$$

We can further rewrite (90) as

$$
\mathbf{w}^{\dagger}=-\frac{v^{*}}{2}\left(\mathbf{R}^{-1} \mathbf{a}_{0}\right)^{\dagger}
$$

Since $\mathbf{R}$ is a Hermitian matrix, i.e., $\mathbf{R}=\mathbf{R}^{\dagger}$, by taking (91) into (92), we have

$$
-\frac{v^{*}}{2}=\frac{1}{\mathbf{a}_{0}^{\dagger} \mathbf{R}^{-1} \mathbf{a}_{0}}
$$

From (92) and (93), the optimal beamforming weights (Vural, 1975; Applebaum, 1976) are therefore given by

$$
\mathbf{w}=\frac{\mathbf{R}^{-1} \mathbf{a}_{0}}{\mathbf{a}_{0}^{\dagger} \mathbf{R}^{-1} \mathbf{a}_{0}}
$$

In general, the corelation matrix $\mathbf{R}=E\left[y(t) y^{*}(t)\right]$ can be approximated by computing the time average of the received signal power over a period of time duration $T$, i.e., $\mathbf{R} \cong(1 / T) \sum_{t=0}^{T-1}|y(t)|^{2}$. It is worthwhile to mention here that for this scheme, the output SNR can be maximized without requiring the knowledge on the directions of the interference sources. Now consider a particular case where only one desired signal source exists and noise is with the covariance matrix $\mathbf{R}_{n}$, i.e., $\mathbf{R}=p_{0} \mathbf{a}_{0} \mathbf{a}_{0}^{\dagger}+\mathbf{R}_{n}$. Use the inversion lemma, we have

$$
\mathbf{R}^{-1}=\mathbf{R}_{n}^{-1}-\frac{p_{0} \mathbf{R}_{n}^{-1} \mathbf{a}_{0} \mathbf{a}_{0}^{\dagger} \mathbf{R}_{n}^{-1}}{1+p_{0} \mathbf{a}_{0}^{\dagger} \mathbf{R}_{n}^{-1} \mathbf{a}_{0}}
$$

After some straightforward manipulation, the optimal beamforming scheme is then degenerated to

$$
\mathbf{w}=\frac{\mathbf{R}^{-1} \mathbf{a}_{0}}{\mathbf{a}_{0}^{\dagger} \mathbf{R}^{-1} \mathbf{a}_{0}}=\frac{\mathbf{R}_{n}^{-1} \mathbf{a}_{0}}{\mathbf{a}_{0}^{\dagger} \mathbf{R}_{n}^{-1} \mathbf{a}_{0}}
$$

In practice, it is hard to know the noise correlation matrix $\mathbf{R}_{n}$; however, it is evident from (96) that one can use $\mathbf{R}$, which could be approximately obtained through the time average of the received signal power, instead of $\mathbf{R}_{n}$ for calculating the optimal beamforming weights in this single-user case. For another special case where noise is spatially white, the optimal beamforming scheme is then further degenerated to the conventional beamforming scheme by substituting $\mathbf{R}_{n}=\sigma_{n}^{2} \mathbf{I}$ into (96):

$$
\mathbf{w}=\frac{\mathbf{R}_{n}^{-1} \mathbf{a}_{0}}{\mathbf{a}_{0}^{\dagger} \mathbf{R}_{n}^{-1} \mathbf{a}_{0}}=\frac{1}{L} \mathbf{a}_{0}
$$




\subsection{Performance of various beamforming techniques}

We show some examples to evaluate the performance of these three beamforming schemes. The number of antenna and the carrier frequency of the sources are set as $L=20$ and $f_{0}=2.3 \mathrm{GHz}$, respectively. The antenna spacing is set to be half the wavelength. The number of sources is given by $M=3$, and the directions of the desired and interfering signals are given by $20^{\circ}, 5^{\circ}$, and $45^{\circ}$, respectively. Fig. 18 compares the angle responses of the conventional and optimal beamforming schemes. It is shown that the optimal beamforming scheme can efectively filter out the two interfering sources at the directions of $5^{\circ}$ and $45^{\circ}$, as compared with the conventional beamforming scheme. Fig. 19 compares the angle response between the null-steering and the optimal beamforming schemes. Although the nullsteering beamforming scheme can form deep nulls in the directions of the interfering sources, the performance of filtering out the interference sources degrades dramatically when there is small discrepancy between the true and esimated directions. In other words, the null-steering scheme requires extremely accurate estimation on the directions of interfering sources. Since the optimal beamforming does not require the knowledge of the directions of interfering sources; in fact, it can be performed by using the received signals to calcuate the correlation matrix in (90) instead, the optimal beamforming has more robust performance than the null-steering one.

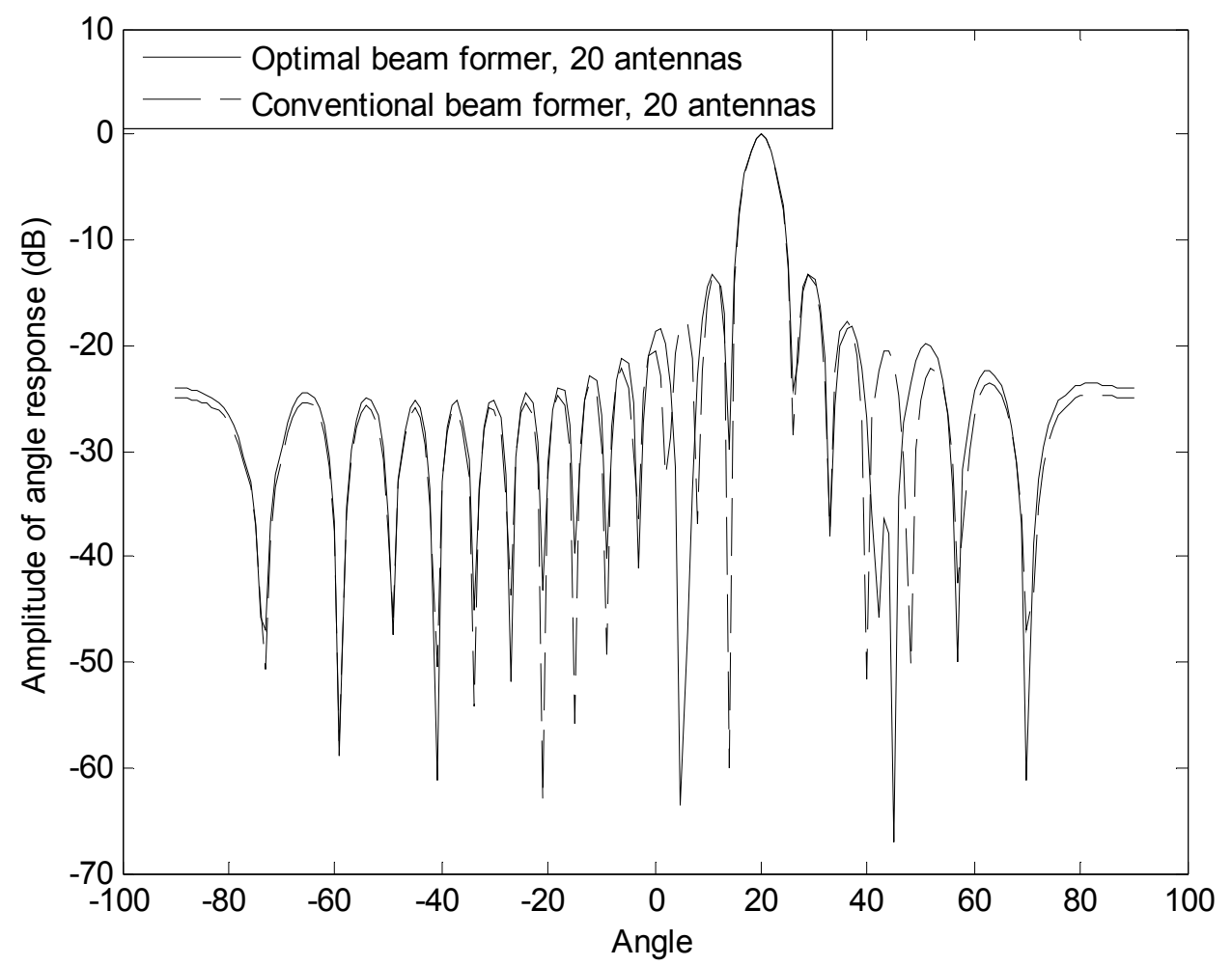

Fig. 18. Angle responses of optimal and conventional beamforming schemes 


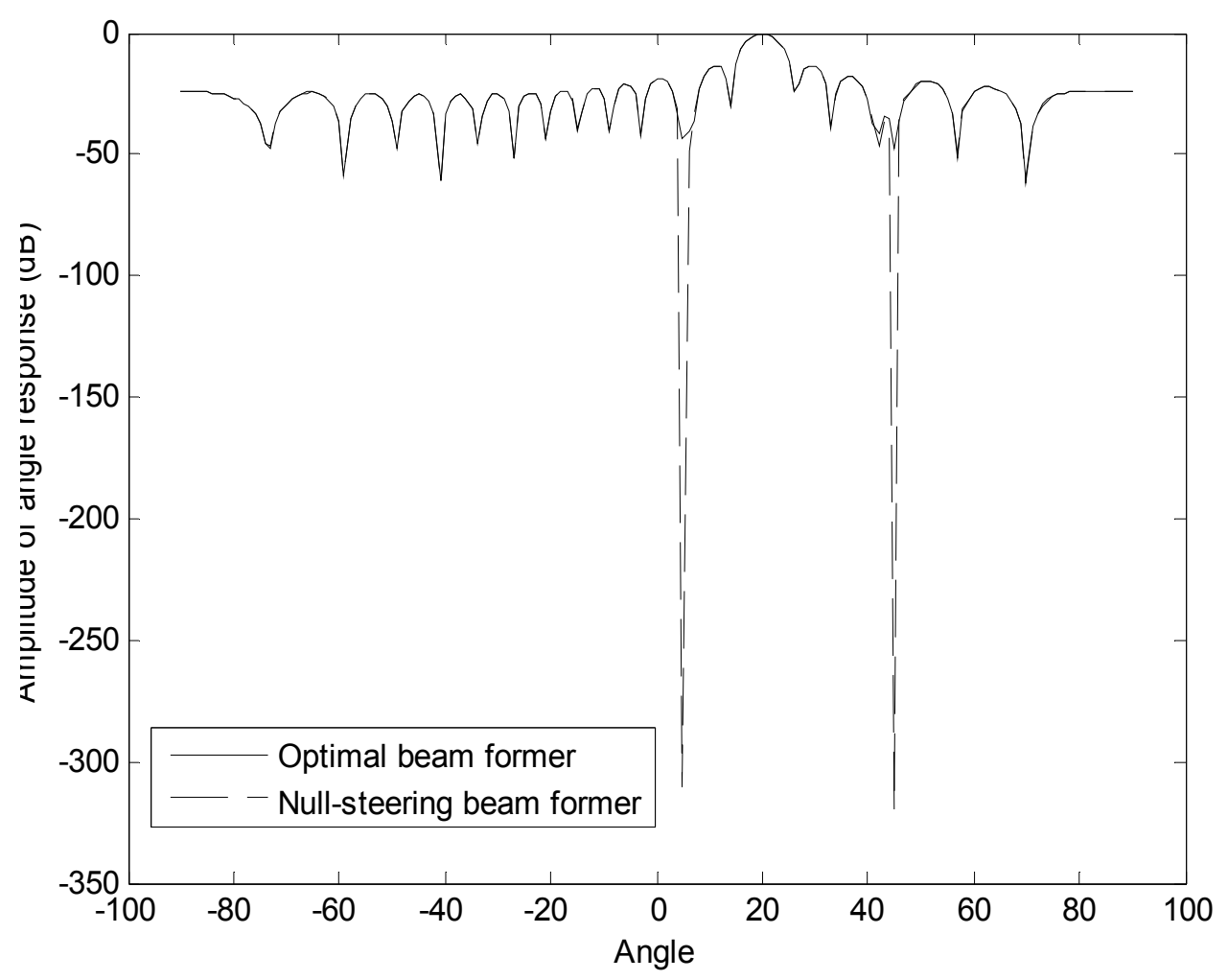

Fig. 19. Angle responses of optimal and null-steering schemes

\section{References}

Abadir, K. M. \& Magnus, J. R. (2006). Matrix Algebra, Cambridge University Press, ISBN 9780-521-53746-9, New York, USA.

Applebaum, S. P. (1976). Adaptive Arrays, IEEE Transactions on Antennas and Propagation, Vol.24, No.5, (Sep 1976), pp.585-598, ISSN: 0018-926X.

D’Assumpcao, H. A. \& Mountford, G. E. (1984). An Overview of Signal Processing for Arrays of Receivers, Journal of the Institution of Engineers (Australia), Vol.4, pp.6-19, ISSN 0020-3319.

Foschini, G. J. (1996). Layered Space-Time Architecture for Wireless Communication in a Fading Environment When Using Multi-Element Antennas. Bell Labs Technical Journal, Vol.1, No.2, (Summer 1999), pp.41-59, ISSN 1538-7305.

Foschini, G. J. \& Gans, M. J. (1998). On Limits of Wireless Communications in a Fading Environment when Using Multiple Antennas. Wireless Personal Communications, Vol.6, No.3, (March 1998), pp.311-335, ISSN 0929-6212.

Foschini, G. J., Golden, G. D., Valenzuela, R. A. \& Wolniansky, P. W. (1999). Simplified Processing for High Spectral Efficiency Wireless Communication Employing Multi- 
Element Arrays. IEEE Journal on Selected Areas in Communications, Vol.17, No.11, (Nov 1999), pp.1841-1852, ISSN 0733-8716.

Giannakis, G. B., Liu, Z., Zhuo, S. \& Ma, X. (2006). Space-Time Coding for Broadband Wireless Communications, John Wiley, ISBN 978-0-471-21479-3, New Jersey, USA.

Godara, L. C. (1997). Application of Antenna Arrays to Mobile Communications. II. BeamForming and Direction-of-Arrival Considerations. Proceedings of IEEE, Vol.85, No.8, (Aug 1997), pp.1195-1245, ISSN 0018-9219.

Ku, M.-L. \& Huang, C.-C. (2006). A Complementary Code Pilot-Based Transmitter Diversity Technique for OFDM Systems. IEEE Transactions on Wireless Communications, Vol. 5, No. 2, (March 2006), pp.504-508, ISSN 1536-1276.

Ku, M.-L. \& Huang, C.-C. (2008). A Refined Channel Estimation Method for STBC/OFDM Systems in High-Mobility Wireless Channels. IEEE Transactions on Wireless Communications, Vol. 7, No. 11, (Nov 2008), pp.4312-4320, ISSN 1536-1276.

Lin, J.-C. (2009). Channel Estimation Assisted by Postfixed Pseudo-Noise Sequences Padded with Zero Samples for Mobile Orthogonal-Frequency-Division-Multiplexing Communications. IET Communications, Vol.3, No.4, (April 2009), pp.561-570, ISSN 1751-8628.

Lin, J.-C. (2009). Least-Squares Channel Estimation Assisted by Self-Interference Cancellation for Mobile Pseudo-Random-Postfix Orthogonal-Frequency-Division Multiplexing Applications. IET Communications, Vol.3, No.12, (Dec 2009), pp.1907-1918, ISSN 1751-8628.

Lo, T. K. Y. (1999). Maximum Ratio Transmission. IEEE Transactions on Communications, Vol. 47, No. 10, (Oct 1999), pp.1458-1461, ISSN 0090-6778.

Rappaport, T. S. (2002). Wireless Communications: Principles and Practice (2nd Edition), Prentice Hall, ISBN 0-13-042232-0, New Jersey, USA.

Simon, M. K. \& Alouini, M. S. (1999). A Unified Performance Analysis of Digital Communication with Dual Selective Combining Diversity over Correlated Rayleigh and Nakagami-m Fading Channels. IEEE Transactions on Communications, Vol.47, No.1, (Jan 1999), pp.33-43, ISSN 0090-6778.

Tarokh, V., Seshadri, N. \& Calderbank, A. R. (1998). Space-Time Codes for High Data Rate Wireless Communication: Performance Criterion and Code Construction. Information Theory. IEEE Transactions on Information Theory, Vol.44, No.2, (Mar 1998), pp.744-765, ISSN 0018-9448.

Tarokh, V., Jafarkhani, H. \& Calderbank, A. R. (1999). Space-Time Block Codes from Orthogonal Designs. IEEE Transactions on Information Theory, Vol.45, No.5, (Jul 1999), pp.1456-1467, ISSN 0018-9448.

Vural, A. M. (1975). An Overview of Adaptive Array Processing for Sonar Application. Proceedings of Electronics and Aerospace Systems Conference, 1975 (EASCON'75), pp.34A-34M.

Wittneben, A. (1993). A New Bandwidth Efficient Transmit Antenna Modulation Diversity Scheme for Linear Digital Modulation. Proceedings of IEEE Communications Conference, pp.1630-1634, ISSN: 0-7803-0950-2, Geneva, Switzerland, May 23-26, 1993. 
Wolniansky, P. W., Foschini, G. J., Golden, G. D. \& Valenzuela, R. A. (1998). V-BLAST: An Architecture for Realizing Very High Data Rates over The Rich-Scattering Wireless Channel. Proceedings of URSI International Symposium on Signals, Systems, and Electronics, pp.295-300, ISSN 0-7803-4900-8, Pisa, Italy, Sep 29-Oct 2, 1998. 


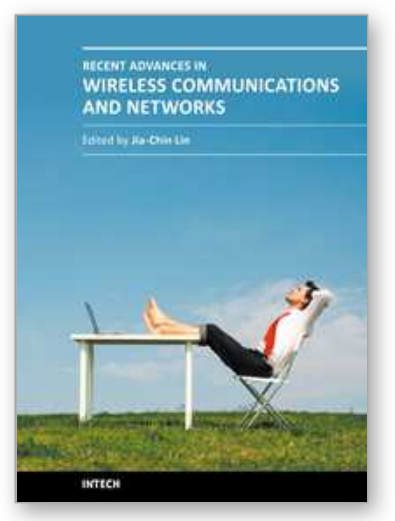

\author{
Recent Advances in Wireless Communications and Networks \\ Edited by Prof. Jia-Chin Lin
}

ISBN 978-953-307-274-6

Hard cover, 454 pages

Publisher InTech

Published online 23, August, 2011

Published in print edition August, 2011

This book focuses on the current hottest issues from the lowest layers to the upper layers of wireless communication networks and provides â€œreal-timeâ€ research progress on these issues. The authors have made every effort to systematically organize the information on these topics to make it easily accessible to readers of any level. This book also maintains the balance between current research results and their theoretical support. In this book, a variety of novel techniques in wireless communications and networks are investigated. The authors attempt to present these topics in detail. Insightful and reader-friendly descriptions are presented to nourish readers of any level, from practicing and knowledgeable communication engineers to beginning or professional researchers. All interested readers can easily find noteworthy materials in much greater detail than in previous publications and in the references cited in these chapters.

\title{
How to reference
}

In order to correctly reference this scholarly work, feel free to copy and paste the following:

Han-Kui Chang, Meng-Lin Ku, Li-Wen Huang and Jia-Chin Lin (2011). Multiple Antenna Techniques, Recent Advances in Wireless Communications and Networks, Prof. Jia-Chin Lin (Ed.), ISBN: 978-953-307-274-6, InTech, Available from: http://www.intechopen.com/books/recent-advances-in-wireless-communications-andnetworks/multiple-antenna-techniques

\section{INTECH}

open science | open minds

\author{
InTech Europe \\ University Campus STeP Ri \\ Slavka Krautzeka 83/A \\ 51000 Rijeka, Croatia \\ Phone: +385 (51) 770447 \\ Fax: +385 (51) 686166 \\ www.intechopen.com
}

\author{
InTech China \\ Unit 405, Office Block, Hotel Equatorial Shanghai \\ No.65, Yan An Road (West), Shanghai, 200040, China \\ 中国上海市延安西路65号上海国际贵都大饭店办公楼 405 单元 \\ Phone: +86-21-62489820 \\ Fax: +86-21-62489821
}


(C) 2011 The Author(s). Licensee IntechOpen. This chapter is distributed under the terms of the Creative Commons Attribution-NonCommercialShareAlike-3.0 License, which permits use, distribution and reproduction for non-commercial purposes, provided the original is properly cited and derivative works building on this content are distributed under the same license. 